\title{
Topology optimization for eigenfrequencies of a rotating thin plate via moving morphable components
}

\author{
Sun, Jialiang; Tian, Qiang; Hu, Haiyan; Pedersen, Niels L.
}

Published in:

Journal of Sound and Vibration

Link to article, DOI:

10.1016/j.jsv.2019.01.054

Publication date:

2019

Document Version

Peer reviewed version

Link back to DTU Orbit

Citation (APA):

Sun, J., Tian, Q., Hu, H., \& Pedersen, N. L. (2019). Topology optimization for eigenfrequencies of a rotating thin plate via moving morphable components. Journal of Sound and Vibration, 448, 83-107.

https://doi.org/10.1016/j.jsv.2019.01.054

\section{General rights}

Copyright and moral rights for the publications made accessible in the public portal are retained by the authors and/or other copyright owners and it is a condition of accessing publications that users recognise and abide by the legal requirements associated with these rights.

- Users may download and print one copy of any publication from the public portal for the purpose of private study or research.

- You may not further distribute the material or use it for any profit-making activity or commercial gain

- You may freely distribute the URL identifying the publication in the public portal

If you believe that this document breaches copyright please contact us providing details, and we will remove access to the work immediately and investigate your claim. 


\title{
Topology optimization for eigenfrequencies of a rotating thin plate via moving morphable components
}

\author{
Jialiang Sun ${ }^{\mathrm{a}}$, Qiang $\operatorname{Tian}^{\mathrm{b}}$, Haiyan $\mathrm{Hu}^{\mathrm{a}, \mathrm{b},{ }^{*}}$, Niels L. Pedersen ${ }^{\mathrm{c}}$ \\ ${ }^{a}$ State Key Laboratory of Mechanics and Control of Mechanical Structures, College of Aerospace \\ Engineering, Nanjing University of Aeronautics and Astronautics, Nanjing 210016, China \\ ${ }^{\mathrm{b}} \mathrm{MOE}$ Key Laboratory of Dynamics and Control of Flight Vehicle, School of Aerospace Engineering, \\ Beijing Institute of Technology, Beijing 100081, China \\ ${ }^{\mathrm{c}}$ Department of Mechanical Engineering, Solid Mechanics, Technical University of Denmark, Kongens \\ Lyngby 2800, Denmark
}

\begin{abstract}
An efficient and explicit topology optimization approach is initially proposed for eigenfrequencies of a rotating thin plate in this study. First of all, an accurate dynamic model of the rotating thin plate is established via the thin plate elements of the absolute nodal coordinate formulation (ANCF). When performing the modal characteristic analysis of the rotating thin plate at a prescribed angular velocity, the linear perturbation analysis is employed, during which the coupling between the membrane and bending deformations is considered. The coupling term makes the dynamic model established in the study more accurate than conventional models, especially in the case of large deformations. Then, the moving morphable components (MMC) are used to describe the topology of the plate. In the frame of MMC-based topology optimization, explicit geometrical parameters, positions, and orientations of the components are taken as the design variables so that the total number of the design variables can be greatly reduced. For the topology optimization, the sensitivities of a simple eigenfrequency and multiply repeated eigenfrequencies with respect to a design variable are analytically derived. During the optimization, in order to remove the localized modes in the low-density areas, the mass and stiffness matrices of the thin plate elements of ANCF are carefully penalized. Finally, four numerical examples are presented to validate the proposed topology optimization approach and to demonstrate its effectiveness for two objectives, i.e., maximizing either the first eigenfrequency or the gap between two consecutive eigenfrequencies of a rotating thin plate.
\end{abstract}

Keywords: Topology optimization; Eigenfrequency; Rotating thin plate; Moving morphable components; Absolute nodal coordinate formulation

\footnotetext{
${ }^{*}$ Corresponding author.

E-mail addresses: sunjialiang@nuaa.edu.cn (J. Sun), tianqiang_hust@aliyun.com (Q. Tian), hhyae@nuaa.edu.cn (H. Hu),nlp@mek.dtu.dk (N.L. Pedersen).
} 


\section{Introduction}

Rotating structures have seen a great variety of applications to industrial products, such as a turbo engine, a helicopter and a spinning solar sail [1-4]. Previous studies have shown that the dynamic characteristics of a rotating structure are more complicated than non-rotating ones because of the centrifugal stiffening effect $[1,3,5$ 7]. For example, the eigenfrequency loci veering, eigenfrequency loci crossing and mode switching phenomena have been observed with an increase of the rotating speed. In practice, the resonance of a rotating structure is usually not desirable and should be avoided by moving the fundamental eigenfrequency of the rotating structure away from the excitation frequency or by enlarging the gap between two consecutive eigenfrequencies to make the eigenfrequency band around the excitation frequency as wide as possible. Hence, the optimization design for the eigenfrequencies of a rotating structure is of great significance in engineering.

Recent years have witnessed the efforts to optimize the eigenfrequencies of a rotating beam with the help of topology optimization by Liu et al. [4]. They optimized the topology of the cross-section of a rotating beam for two objectives, i.e., maximizing either the fundamental eigenfrequency or the gap between two consecutive eigenfrequencies. For the plate-like structures with low aspect ratios, such as a spinning solar sail, however, the model of a rotating beam is not accurate enough. Hence, the model of a rotating thin plate instead should be established [3]. Compared with a rotating beam, the topology optimization for eigenfrequencies of a rotating thin plate definitely faces more tough challenges, including the accurate modeling scheme, the efficient topology optimization solution, the avoidance of localized modes and the sensitivity analysis.

Many researchers have studied the dynamic models and modal characteristics of a rotating plate [3, 7-12] since the 1970s. However, Zhao et al. [3] pointed out that the conventional dynamic models [10-12] of a rotating plate may encounter numerical problems associated with the coupling of large rotations and large deformations. This is due to that those models [10-12] used the infinitesimal rotations as the nodal coordinates. Zhao et al. [3] established a more accurate model for a rotating thin plate via the absolute nodal coordinate formulation (ANCF). As a nonincremental finite element method, the ANCF uses global positions and slopes as the nodal coordinates in a global frame of coordinates [13]. As a result, the modeling scheme of ANCF for a rotating structure has many merits, such as the elimination of coordinate transformation, the constant mass matrix and the very simple inertia forces [1, 3, 7]. Zhang et al. [1] pointed out that when a rotating structure is undergoing a small deformation, the modal analysis based on the ANCF model and the conventional models agree with each other very well. When the deformation of a rotating structure is large, however, their differences in the modal characteristics become obvious. Zhao et al. [3] also reported this phenomenon as the modeling scheme of ANCF showed a higher accuracy than the conventional models when the longitudinal deformation of a rotating thin plate was large.

When performing the modal characteristic analysis of the rotating thin plate at a 
particular static equilibrium configuration and a prescribed angular velocity, the linear perturbation analysis is used and the coupling between the membrane and bending deformations is taken into consideration. The nonlinear dynamic equations are linearized at the equilibrium configuration and the resulting linear dynamic equations determine the eigenvalues and eigenvectors of the system [14]. Such an analysis process can be used to study the system stability [15], which is not in the scope of this study. For the modal analysis of the rotating thin plate, the equilibrium configuration is first obtained from the nonlinear static analysis. Then, at the particular equilibrium configuration, the amplitude of the vibration of the thin plate is assumed to be small. Hence, linearization is only used for modal analysis. The dynamic model of the rotating thin plate is nonlinear and takes the coupling between the membrane and bending deformations into consideration. Besides, in the field of multibody system dynamics, the frequencies of the linear problem have no effect on the frequencies of the solution of the nonlinear problem [16].

The topology optimization for eigenfrequencies of a non-rotating plate has attracted continuous attention [17-22] since the pioneering work of Bendsøe and Kikuchi [23]. Nevertheless, no studies have been reported so far for the topology optimization for eigenfrequencies of a rotating thin plate. Most of the previous topology optimization approaches are based on the variable densities [19-21] or level set functions [24], which may contain many design variables, especially for large-scale problems. A large number of design variables will increase the computation cost associated with the sensitivity analysis and optimization solution. Ferrari et al. [21] tried to use efficient solution techniques to solve this problem. To reduce the number of the design variables, Guo et al. [25] proposed the method of moving morphable components (MMC) to simplify the structure to be designed by using the explicit geometrical sizes, positions, and orientations of the components as the design variables. This method has seen many applications [26-28], but not yet to the topology optimization for the eigenfrequencies of any rotating structures.

For the topology optimization for eigenfrequencies of plates, the localized modes may occur in the low-density areas [20]. This is due to the fact that the low-density areas are so flexible that they may control the lowest eigenfrequency of the entire structure. The localized modes are, therefore, a kind of fake modes and need to be avoided during the topology optimization process. To remove the localized modes, many methods have been proposed [20, 21, 29, 30]. As a matter of fact, these methods are essentially the same by modifying the ratio between the penalization of the mass and stiffness or by penalizing the mass harder for the finite elements in low-density areas so as to move the localized modes to higher orders. For a rotating thin plate, however, with an increase of the angular velocity, the eigenfrequencies also increase, which may result in the failure for removing the localized modes at a high angular velocity. Hence, the penalization of the mass and stiffness need to be carefully dealt with for a rotating thin plate. The other important issue about the topology optimization for eigenfrequencies is the sensitivity analysis of eigenfrequencies, especially of multiply repeated eigenfrequencies, with respect to a design variable. Last decades have witnessed the development of many methods for the sensitivity analysis of 
eigenfrequencies, for example, the methods by computing the sensitivities of eigenvalues [19, 31] and the methods via a smooth approximation [4, 32-34].

In this study, a new topology optimization approach based on MMC is proposed for the eigenfrequencies of a rotating thin plate modeled via the ANCF under two objectives. One is to maximize the first eigenfrequency and the other is to maximize the gap between two consecutive eigenfrequencies. In order to avoid that all the masses move to one side of the rotating thin plate when maximizing the first eigenfrequency, some nonstructural masses are assumed in the design domain.

The rest of the paper is organized as follows. In Section 2, the dynamic model of a rotating thin plate with a nonstructural mass at the tip is established via the thin plate element of ANCF. In Section 3, the dynamic equation and its dimensionless form for modal analysis of the rotating thin plate are derived. In Section 4, the basic ideas of the MMC are revisited, the penalization of the mass and stiffness is defined, and two optimization formulations are outlined. In Section 5, the sensitivities of a simple eigenfrequency and multiply repeated eigenfrequencies with respect to a design variable are presented in detail. In Section 6, four numerical examples are provided to validate the approach. Finally, some concluding remarks are drawn in Section 7.

\section{Dynamic model}

\subsection{Physical description}

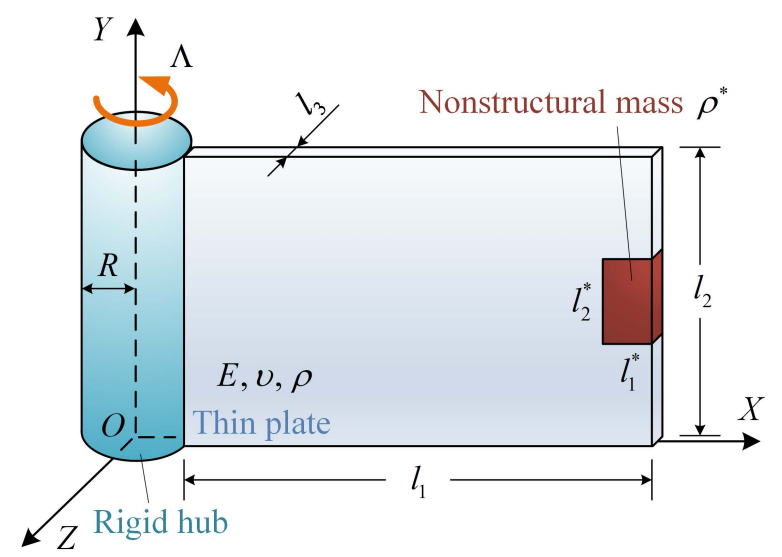

Fig. 1. A rotating thin plate with a nonstructural mass at the tip edge.

In this study, the dynamics and optimization of a rectangular thin plate attached to a rigid hub is considered, as shown in Fig. 1. The rotation axis is parallel to one of the edges of the plate and the angular velocity is $\Lambda$. The radius of the rigid hub is $R$. The length, width, and thickness of the thin plate are $l_{1}, l_{2}$, and $l_{3}$, respectively. To avoid all the masses moving to the fixed edge of the thin plate when maximizing its first eigenfrequency, the non-design domain with a so-called nonstructural mass is imposed at the tip edge of the plate. The nonstructural mass which is centered vertically has a length of $l_{1}^{*}$ and a width of $l_{2}^{*}$, as shown in Fig. 1. E and $v$ represent Young's modulus and Poisson's ratio of the plate material. $\rho$ and $\rho^{*}$ are the material densities of the solid domain and the nonstructural mass, respectively. 


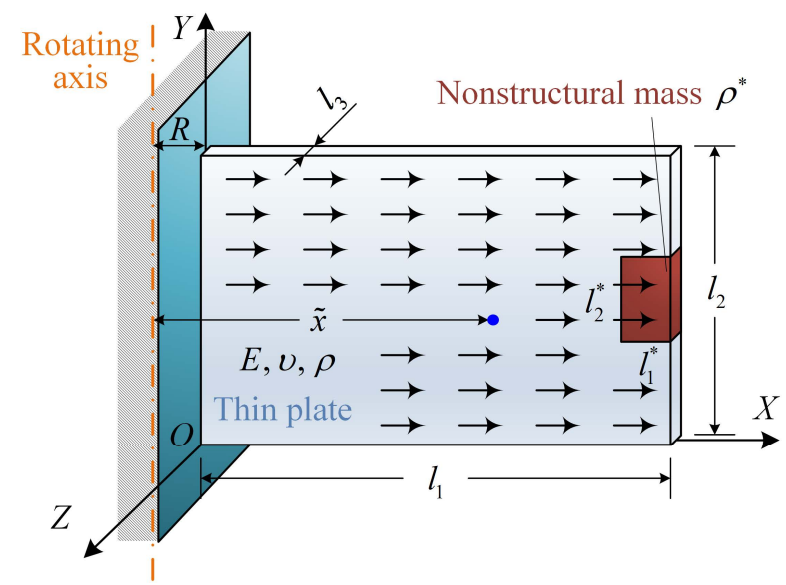

Fig. 2. The cantilever thin plate with a nonstructural mass subject to equivalent forces.

For the modal characteristic analysis, the rotating thin plate can be equivalently treated as a non-rotating cantilever thin plate, as shown in Fig. 2, subject to the corresponding centrifugal forces. Therefore, the focus of this study is to accurately compute the elastic forces and the equivalent centrifugal forces at the deformed configuration and then to obtain the tangent stiffness matrix for modal analysis and optimization design. The dynamic model of the rotating thin plate is the same as that of Zhao et al. [3]. Zhao et al. [3] not only derived the analytical expressions of elastic forces and their Jacobians, but also validated the dynamic model via three classic examples. In this section, the dynamic model of a rotating thin plate is briefly introduced.

\subsection{Implementation of a thin plate element of ANCF}

To establish the dynamic model of the cantilever thin plate with a nonstructural mass in Fig. 2, the rectangular thin plate element of ANCF [3, 35] is employed, as shown in Fig. 3. The rectangular thin plate element uses the global positions $\mathbf{r}_{i}$ and in-plane slopes $\mathbf{r}_{i, x}$ and $\mathbf{r}_{i, y}$ of four nodes $(i=1,2, \cdots, 4)$ as the generalized coordinates $\mathbf{e}=\left[\begin{array}{lllllll}\mathbf{r}_{1}^{\mathrm{T}} & \mathbf{r}_{1, x}^{\mathrm{T}} & \mathbf{r}_{1, y}^{\mathrm{T}} & \cdots & \mathbf{r}_{4}^{\mathrm{T}} & \mathbf{r}_{4, x}^{\mathrm{T}} & \mathbf{r}_{4, y}^{\mathrm{T}}\end{array}\right]^{\mathrm{T}}$, where $\mathbf{r}_{i, x}=\partial \mathbf{r}_{i} / \partial x$.

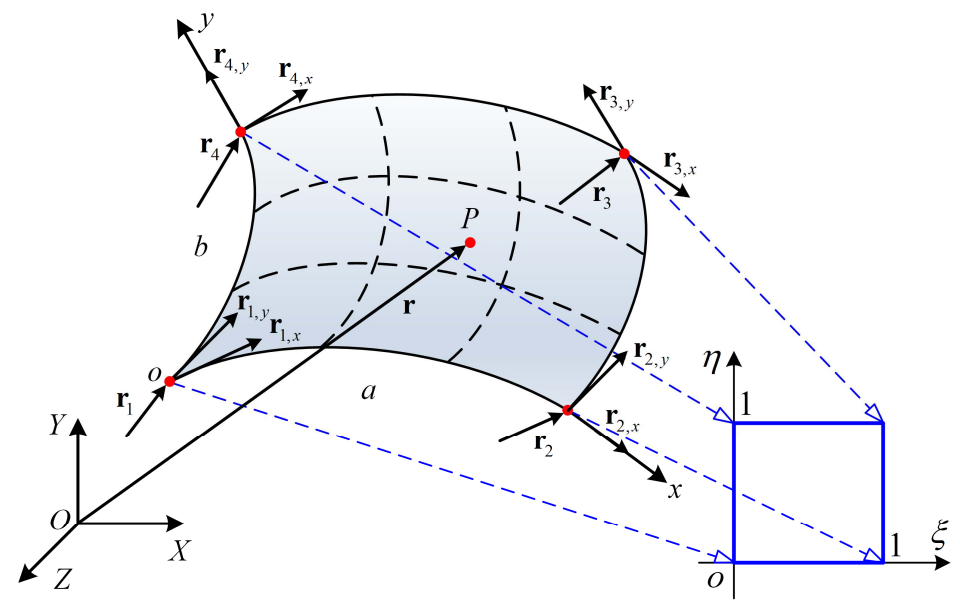

Fig. 3. A deformed rectangular thin plate element of ANCF. 
The position vector $\mathbf{r}$ of an arbitrary point $P$ in the global frame of coordinates $O-X Y Z$ can be defined by the Hermite interpolation as $\mathbf{r}=\mathbf{S}(\xi, \eta) \mathbf{e}$, where $\mathbf{S}=\left[\begin{array}{llll}S_{1} \mathbf{I} & S_{2} \mathbf{I} & \cdots & S_{12} \mathbf{I}\end{array}\right]$ is the shape function matrix with $\mathbf{I}$ denoting the $3 \times 3$ identity matrix. The entries of $\mathbf{S}$ are defined as follows

$$
\left\{\begin{array}{l}
S_{1}=-(\xi-1)(\eta-1)\left(2 \eta^{2}-\eta+2 \xi^{2}-\xi-1\right), S_{2}=-a \xi(\xi-1)^{2}(\eta-1) \\
S_{3}=-b \eta(\eta-1)^{2}(\xi-1), S_{4}=\xi\left(2 \eta^{2}-\eta-3 \xi+2 \xi^{2}\right)(\eta-1), \\
S_{5}=-a \xi^{2}(\xi-1)(\eta-1), S_{6}=b \xi \eta(\eta-1)^{2} \\
S_{7}=-\xi \eta\left(1-3 \xi-3 \eta+2 \eta^{2}+2 \xi^{2}\right), S_{8}=a \xi^{2} \eta(\xi-1), \\
S_{9}=b \xi \eta^{2}(\eta-1), S_{10}=\eta(\xi-1)\left(2 \xi^{2}-\xi-3 \eta+2 \eta^{2}\right), \\
S_{11}=a \xi \eta(\xi-1)^{2}, S_{12}=-b \eta^{2}(\xi-1)(\eta-1),
\end{array}\right.
$$

where $\xi=x / a, \eta=y / b, x$ and $y$ are the local coordinates, $a$ and $b$ are the undeformed length and width of the thin plate element, respectively.

\subsection{Mass matrix of a thin plate element}

The kinetic energy of the $e$-th thin plate element is

$$
T=\frac{1}{2} \int_{V_{0}} \rho \dot{\mathbf{r}}^{\mathrm{T}} \mathbf{r} \mathrm{d} V=\frac{1}{2} \dot{\mathbf{e}}^{\mathrm{T}} \mathbf{M}_{e} \dot{\mathbf{e}}
$$

where $\mathbf{M}_{e}$ is the mass matrix of the element defined as follows

$$
\mathbf{M}_{e}=\int_{V_{0}} \rho \mathbf{S}(\xi, \eta)^{\mathrm{T}} \mathbf{S}(\xi, \eta) \mathrm{d} V=a b c \rho \int_{0}^{1} \int_{0}^{1} \mathbf{S}(\xi, \eta)^{\mathrm{T}} \mathbf{S}(\xi, \eta) \mathrm{d} \xi \mathrm{d} \eta .
$$

Likewise, the mass matrix of the $e$-th thin plate element for the nonstructural mass is

$$
\mathbf{M}_{e}^{*}=a b c \rho^{*} \int_{0}^{1} \int_{0}^{1} \mathbf{S}(\xi, \eta)^{\mathrm{T}} \mathbf{S}(\xi, \eta) \mathrm{d} \xi \mathrm{d} \eta .
$$

In Eqs. (3) and (4), $c$ is the thickness of the thin plate element, i.e., $c=l_{3}$.

\subsection{Generalized elastic forces of a thin plate element}

The strain energy of the $e$-th thin plate element is

$$
U=U^{\varepsilon}+U^{\kappa}=\frac{1}{2} \int_{V_{0}} \boldsymbol{\varepsilon}^{\mathrm{T}} \mathbf{E}^{\varepsilon} \boldsymbol{\varepsilon} \mathrm{d} V+\frac{1}{2} \int_{V_{0}} \boldsymbol{\kappa}^{\mathrm{T}} \mathbf{E}^{\kappa} \boldsymbol{\kappa} \mathrm{d} V
$$

where $U^{\varepsilon}$ is the strain energy due to the longitudinal and shear deformations, and $U^{\kappa}$ is the term associated with the bending and twist deformations. Under the assumption of plane stress, the elastic coefficient matrices $\mathbf{E}^{\varepsilon}$ and $\mathbf{E}^{\kappa}$ are defined as follows

$$
\mathbf{E}^{\varepsilon}=\frac{E}{1-v^{2}} \mathbf{E}=\frac{E}{1-v^{2}}\left[\begin{array}{ccc}
1 & v & 0 \\
v & 1 & 0 \\
0 & 0 & \frac{1-v}{2}
\end{array}\right], \mathbf{E}^{\kappa}=\frac{c^{2}}{12} \mathbf{E}^{\varepsilon}=\frac{E c^{2}}{12\left(1-v^{2}\right)} \mathbf{E},
$$

In Eq. (5), the strain vector $\boldsymbol{\varepsilon}$ at the midsurface and the curvature vector $\boldsymbol{\kappa}$ of the plate yield 


$$
\boldsymbol{\varepsilon}=\left[\begin{array}{lll}
\frac{1}{2}\left(\mathbf{r}_{, x}^{\mathrm{T}} \mathbf{r}_{, x}-1\right) & \frac{1}{2}\left(\mathbf{r}_{, y}^{\mathrm{T}} \mathbf{r}_{, y}-1\right) & \mathbf{r}_{, x}^{\mathrm{T}} \mathbf{r}_{, y}
\end{array}\right]^{\mathrm{T}}, \boldsymbol{\kappa}=\left[\begin{array}{lll}
\frac{\mathbf{r}_{, x x}^{\mathrm{T}} \mathbf{n}}{\|\mathbf{n}\|^{3}} & \frac{\mathbf{r}_{, y y}^{\mathrm{T}} \mathbf{n}}{\|\mathbf{n}\|^{3}} & \frac{2 \mathbf{r}_{, x y}^{\mathrm{T}} \mathbf{n}}{\|\mathbf{n}\|^{3}}
\end{array}\right]^{\mathrm{T}},
$$

where $\mathbf{r}_{, x x}=\partial^{2} \mathbf{r} / \partial x^{2}$ and $\mathbf{n}=\mathbf{r}_{, x} \times \mathbf{r}_{, y}$.

The vector of generalized elastic forces of the thin plate element can then be expressed as

$$
\mathbf{F}_{e}=\left(\frac{\partial U^{\varepsilon}}{\partial \mathbf{e}}\right)^{\mathrm{T}}+\left(\frac{\partial U^{\kappa}}{\partial \mathbf{e}}\right)^{\mathrm{T}}=\mathbf{F}_{e}^{\varepsilon}+\mathbf{F}_{e}^{\kappa}
$$

where

$$
\begin{aligned}
& \mathbf{F}_{e}^{\varepsilon}=\int_{V_{0}}\left(\frac{\partial \boldsymbol{\varepsilon}}{\partial \mathbf{e}}\right)^{\mathrm{T}} \mathbf{E}^{\varepsilon} \boldsymbol{\varepsilon} \mathrm{d} V=\frac{a b c E}{1-v^{2}} \int_{0}^{1} \int_{0}^{1}\left(\frac{\partial \boldsymbol{\varepsilon}}{\partial \mathbf{e}}\right)^{\mathrm{T}} \mathbf{E} \boldsymbol{\varepsilon} \mathrm{d} \xi \mathrm{d} \eta, \\
& \mathbf{F}_{e}^{\kappa}=\int_{V_{0}}\left(\frac{\partial \mathbf{\kappa}}{\partial \mathbf{e}}\right)^{\mathrm{T}} \mathbf{E}^{\kappa} \mathbf{\kappa} \mathrm{d} V=\frac{a b c^{3} E}{12\left(1-v^{2}\right)} \int_{0}^{1} \int_{0}^{1}\left(\frac{\partial \mathbf{\kappa}}{\partial \mathbf{e}}\right)^{\mathrm{T}} \mathbf{E} \boldsymbol{\kappa} \mathrm{d} \xi \mathrm{d} \eta,
\end{aligned}
$$

The Jacobian of the vector of generalized elastic forces satisfies

$$
\frac{\partial \mathbf{F}_{e}}{\partial \mathbf{e}}=\frac{\partial \mathbf{F}_{e}^{\varepsilon}}{\partial \mathbf{e}}+\frac{\partial \mathbf{F}_{e}^{\kappa}}{\partial \mathbf{e}}
$$

The readers can refer to [3] for the efficient formulae of the elastic forces and the corresponding Jacobian formulations.

\subsection{Generalized centrifugal forces of a thin plate element}

The virtual work of the centrifugal forces on the $e$-th thin plate element can be written as

$$
\delta W=\int_{V_{0}}\left[\begin{array}{lll}
\rho \Lambda^{2} \tilde{x} & 0 & 0
\end{array}\right] \delta \mathbf{r d} V=\int_{V_{0}}\left[\begin{array}{lll}
\rho \Lambda^{2} \tilde{x} & 0 & 0
\end{array}\right] \mathbf{S}(\xi, \eta) \mathrm{d} V \delta \mathbf{e}=\mathbf{Q}_{e}{ }^{\mathrm{T}} \delta \mathbf{e},
$$

where $\tilde{X}$ denotes the distance between the integral point and the rotating axis, as shown in Fig. 2. The vector of generalized centrifugal forces can then be expressed as

$$
\mathbf{Q}_{e}=a b c \rho \Lambda^{2} \int_{0}^{1} \int_{0}^{1} \mathbf{S}(\xi, \eta)^{\mathrm{T}} \tilde{\mathbf{S}}(\xi, \eta) \mathrm{d} \xi \mathrm{d} \eta \mathbf{e}+a b c \rho \Lambda^{2} R \int_{0}^{1} \int_{0}^{1} \mathbf{S}_{1}(\xi, \eta)^{\mathrm{T}} \mathrm{d} \xi \mathrm{d} \eta .
$$

Accordingly, the vector of generalized centrifugal forces of the $e$-th thin plate element for the nonstructural mass is

$$
\mathbf{Q}_{e}^{*}=a b c \rho^{*} \Lambda^{2} \int_{0}^{1} \int_{0}^{1} \mathbf{S}(\xi, \eta)^{\mathrm{T}} \tilde{\mathbf{S}}(\xi, \eta) \mathrm{d} \xi \mathrm{d} \eta \mathbf{e}+a b c \rho^{*} \Lambda^{2} R \int_{0}^{1} \int_{0}^{1} \mathbf{S}_{1}(\xi, \eta)^{\mathrm{T}} \mathrm{d} \xi \mathrm{d} \eta .
$$

In Eqs. (13) and (14), $\tilde{\mathbf{S}}=\left[\begin{array}{llll}S_{1} \tilde{\mathbf{I}} & S_{2} \tilde{\mathbf{I}} & \cdots & S_{12} \tilde{\mathbf{I}}\end{array}\right]$ and $\mathbf{S}_{1}=\left[\begin{array}{lllll}S_{1} \mathbf{I}_{1} & S_{2} \mathbf{I}_{1} & \cdots & S_{12} \mathbf{I}_{1}\end{array}\right]$ with $\tilde{\mathbf{I}}=\left[\begin{array}{lll}1 & 0 & 0 \\ 0 & 0 & 0 \\ 0 & 0 & 0\end{array}\right]$ and $\mathbf{I}_{1}=\left[\begin{array}{lll}1 & 0 & 0\end{array}\right]$.

The Jacobian of the vectors of generalized centrifugal forces can be expressed as 


$$
\begin{aligned}
& \frac{\partial \mathbf{Q}_{e}}{\partial \mathbf{e}}=a b c \rho \Lambda^{2} \int_{0}^{1} \int_{0}^{1} \mathbf{S}(\xi, \eta)^{\mathrm{T}} \tilde{\mathbf{S}}(\xi, \eta) \mathrm{d} \xi \mathrm{d} \eta, \\
& \frac{\partial \mathbf{Q}_{e}^{*}}{\partial \mathbf{e}}=a b c \rho^{*} \Lambda^{2} \int_{0}^{1} \int_{0}^{1} \mathbf{S}(\xi, \eta)^{\mathrm{T}} \tilde{\mathbf{S}}(\xi, \eta) \mathrm{d} \xi \mathrm{d} \eta .
\end{aligned}
$$

\section{Modal analysis}

\subsection{Dynamic equation}

The dynamic equation of the undamped cantilever thin plate shown in Fig. 2 can reads

$$
\mathbf{M} \ddot{\mathbf{q}}+\mathbf{F}(\mathbf{q})=\mathbf{Q}(\mathbf{q}),
$$

where $\mathbf{M}$ is the constant mass matrix of the system, $\mathbf{q}$ is the vector of generalized coordinates of the system, $\mathbf{F}(\mathbf{q})$ and $\mathbf{Q}(\mathbf{q})$ are the vectors of generalized elastic forces and the generalized centrifugal forces of the system, respectively. According to Eqs. (3), (4), (8), (13) and (14), if an $N X \times N Y$ mesh size is used to discretize the equivalent cantilever thin plate by using the rectangular thin plate element of ANCF, $\mathbf{M}, \mathbf{F}(\mathbf{q})$ and $\mathbf{Q}(\mathbf{q})$ can be assembled as follows

$$
\left\{\begin{array}{l}
\mathbf{M}=\sum_{e=1}^{N E 1} \mathbf{B}_{e}^{\mathrm{T}} \mathbf{M}_{e} \mathbf{B}_{e}+\sum_{e=1}^{N E 2} \mathbf{B}_{e}^{\mathrm{T}} \mathbf{M}_{e}^{*} \mathbf{B}_{e}, \\
\mathbf{F}(\mathbf{q})=\sum_{e=1}^{N E} \mathbf{B}_{e}^{\mathrm{T}} \mathbf{F}_{e}, \\
\mathbf{Q}(\mathbf{q})=\sum_{e=1}^{N E 1} \mathbf{B}_{e}^{\mathrm{T}} \mathbf{Q}_{e}+\sum_{e=1}^{N E 2} \mathbf{B}_{e}^{\mathrm{T}} \mathbf{Q}_{e}^{*},
\end{array}\right.
$$

with $\mathbf{B}_{e}$ denoting the element transformation matrix by means of $\mathbf{e}=\mathbf{B}_{e} \mathbf{q} . N E 1$ and NE2 denote the total numbers of thin plate elements of ANCF for the design domain and the nonstructural mass, respectively. Obviously, the total number of finite elements is $N E=N E 1+N E 2=N X \times N Y$.

In order to obtain the solution of the eigenvalue problem of the rotating thin plate including the effect of the centrifugal forces, the dynamic equation formulated in Eq. (17) is linearized at a particular static equilibrium configuration $\mathbf{q}$ and a prescribed angular velocity $\Lambda$. There follows the perturbation form of the dynamic equation of the system as below

$$
\mathbf{M} \delta \ddot{\mathbf{q}}+\mathbf{K}(\mathbf{q}) \delta \mathbf{q}=\mathbf{0},
$$

where $\mathbf{K}(\mathbf{q})$ is the tangent stiffness matrix of the system, which is a matrix function of the static equilibrium configuration $\mathbf{q}$ determined by solving the static equilibrium equation as follows

$$
\mathbf{F}(\mathbf{q})-\mathbf{Q}(\mathbf{q})=\mathbf{0}
$$


Thus, the tangent stiffness matrix of the system can be expressed as

$$
\mathbf{K}(\mathbf{q})=\frac{\partial \mathbf{F}}{\partial \mathbf{q}}-\frac{\partial \mathbf{Q}}{\partial \mathbf{q}}=\sum_{e=1}^{N E} \mathbf{B}_{e}^{\mathrm{T}} \frac{\partial \mathbf{F}_{e}}{\partial \mathbf{e}} \mathbf{B}_{e}-\sum_{e=1}^{N E 1} \mathbf{B}_{e}^{\mathrm{T}} \frac{\partial \mathbf{Q}_{e}}{\partial \mathbf{e}} \mathbf{B}_{e}-\sum_{e=1}^{N E 2} \mathbf{B}_{e}^{\mathrm{T}} \frac{\partial \mathbf{Q}_{e}^{*}}{\partial \mathbf{e}} \mathbf{B}_{e} .
$$

The general solution of Eq. (19) can be assumed as $\delta \mathbf{q}=\mathbf{Z} \mathrm{e}^{\mathrm{j} \omega t}$. The substitution of the solution into Eq. (19) leads to the generalized eigenvalue problem

$$
\left(\mathbf{K}-\omega_{n}^{2} \mathbf{M}\right) \mathbf{Z}_{n}=\mathbf{0}\left(n=1,2, \cdots, N_{d}\right) .
$$

where $N_{d}$ is the number of degrees of freedom of the system. The eigenfrequencies $\omega_{n}$ $\left(n=1,2, \cdots, N_{d}\right)$ and the corresponding eigenvectors $\mathbf{Z}_{n} \quad\left(n=1,2, \cdots, N_{d}\right)$ can then be determined by solving Eq. (22) with the subspace iteration method [36, 37].

\subsection{Dimensionless form}

To transfer the dynamic equation (17) into a dimensionless form, the following dimensionless variables and parameters are introduced

$$
\alpha=\frac{\rho^{*}}{\rho}, \beta=\frac{l_{1}}{l_{2}}, \mu=\frac{l_{1}}{l_{3}}, \sigma=\frac{R}{l_{1}}, \gamma=\Lambda T_{0}, \tau=\frac{t}{T_{0}},
$$

where $\alpha, \beta, \mu$, and $\sigma$ represent the nonstructural mass density ratio, the length-width ratio of plate, the length-thickness ratio of plate, and the hub radius ratio, respectively. $\gamma$ is the dimensionless angular velocity of the rigid hub and $\tau$ is the dimensionless time with $T_{0}=\sqrt{\frac{12\left(1-v^{2}\right) \rho l_{1}^{4}}{E l_{3}^{2}}}$.

The dimensionless form of the dynamic equation in Eq. (17) can be expressed as

$$
\overline{\mathbf{M}} \frac{\mathrm{d}^{2} \overline{\mathbf{q}}}{\mathrm{d} \tau^{2}}+\overline{\mathbf{F}}(\overline{\mathbf{q}})=\overline{\mathbf{Q}}(\overline{\mathbf{q}})
$$

where the dimensionless terms $\overline{\mathbf{M}}, \overline{\mathbf{q}}, \overline{\mathbf{F}}(\overline{\mathbf{q}})$, and $\overline{\mathbf{Q}}(\overline{\mathbf{q}})$ are listed in the "Appendix".

The dimensionless form of the eigenvalue problem in Eq. (22) can also be expressed as

$$
\left(\overline{\mathbf{K}}-\bar{\omega}_{n}^{2} \overline{\mathbf{M}}\right) \mathbf{Z}_{n}=\mathbf{0}\left(n=1,2, \cdots, N_{d}\right),
$$

where $\bar{\omega}=\omega T_{0}$ is the dimensionless eigenfrequency with $\bar{\omega}_{n} \quad\left(n=1,2, \cdots, N_{d}\right)$ denoting the dimensionless eigenfrequency of order $n$. The dimensionless tangent stiffness matrix $\overline{\mathbf{K}}(\overline{\mathbf{q}})$ is also listed in the "Appendix".

\section{Topology optimization}

\subsection{Topology description via MMC}

To describe the topology of a rotating thin plate, the quadratically thicknessvarying structural components $[27,38]$ are used as the basic building blocks. As shown 
in Fig. 4, the topology description function (TDF) of the $j$-th component can be mathematically expressed as $[27,38,39]$

$$
\chi_{j}(X, Y)=1-\left(\frac{x}{L}\right)^{p}-\left(\frac{y}{W(x)}\right)^{p},
$$

where $j=1,2, \cdots, N_{c}$ and $N_{c}$ is the number of the structural components. $p$ is a positive even integer, given for instance as $p=6 . L$ and $W(x)$ are the semilength and semiwidth profile of the component, respectively. The semiwidth profile yields the quadratic relation as follows

$$
W(x)=\frac{C_{1}+C_{2}-2 c_{3}}{2 L^{2}} x^{2}+\frac{C_{2}-c_{1}}{2 L} x+C_{3} .
$$

Here, the parameters $c_{1}, c_{2}$, and $c_{3}$ are the semiwidths at both ends and in the middle of the structural component, as illustrated in Fig. 4.

In Fig. 4, $O-X Y$ and $o-x y$ denote the global frame of coordinates and the local frame of coordinates, respectively. The relationship between the local coordinates $(x, y)$ and the global coordinates $(X, Y)$ reads

$$
\left[\begin{array}{l}
x \\
y
\end{array}\right]=\left[\begin{array}{cc}
\cos \theta & \sin \theta \\
-\sin \theta & \cos \theta
\end{array}\right]\left[\begin{array}{c}
X-X_{0} \\
Y-Y_{0}
\end{array}\right],
$$

where $\left(X_{0}, Y_{0}\right)$ is the global coordinates of the component center and $\theta$ is the inclined angle with respect to the $O X$ axis of the component.

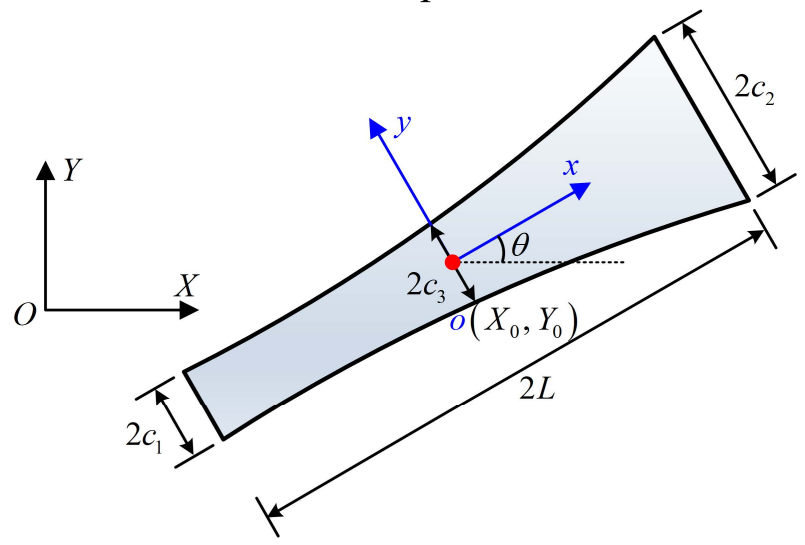

Fig. 4. A two-dimensional thickness-varying structural component.

According to Eqs. (26), (27) and (28), the vector of the design variables for the $j$-th component can be defined as $\mathbf{b}_{j}=\left[\begin{array}{lllllll}X_{0} & Y_{0} & \theta & L & c_{1} & c_{2} & c_{3}\end{array}\right]^{\mathrm{T}}$. Once $\mathbf{b}_{j}$ is obtained, the position, orientation, and sizes of the $j$-th component can be determined. Then, the material domain $\Omega_{j}$ occupied by the $j$-th component, the material interface $\partial \Omega_{j}$ of the $j$-th component, and the void domain $D \backslash \Omega_{j}$ corresponding to the $j$-th component can be given as 


$$
\left\{\begin{array}{l}
\chi_{j}(\mathbf{r})>0 \Leftrightarrow \mathbf{r} \in \Omega_{j} \\
\chi_{j}(\mathbf{r})=0 \Leftrightarrow \mathbf{r} \in \partial \Omega_{j} \\
\chi_{j}(\mathbf{r})<0 \Leftrightarrow \mathbf{r} \in D \backslash \Omega_{j}
\end{array}\right.
$$

where $\mathbf{r}$ is an arbitrary point in the design domain $D$ of $\mathbb{R}^{2}, \chi_{j}$ is the TDF defined in Eq. (26) of the $j$-th component. The TDF $\chi$ and the material domain $\Omega$ of the entire structure to be optimized can be written as

$$
\chi=\max \left(\chi_{1}, \chi_{2}, \cdots, \chi_{N_{c}}\right), \Omega=\bigcup_{j=1}^{N_{c}} \Omega_{j} .
$$

\subsection{Penalization schemes of mass and stiffness}

Based on a fixed ANCF mesh, the physical properties e.g. mass and stiffness of a thin plate element of ANCF become functions of the volume fraction $\rho_{e}$ of the element during the optimization. The volume fraction $\rho_{e}(e=1,2, \cdots, N E 1)$ of the solid material occupied by the $e$-th element can be computed according to the TDF $\chi$ in Eq. (30) as follows [27, 40]

$$
\rho_{e}=\frac{1}{4} \sum_{i=1}^{4} H\left(\chi\left(\mathbf{r}_{i}^{e}\right)\right),
$$

where $\mathbf{r}_{i}^{e} \quad(i=1, \cdots, 4)$ are the position vectors of the four nodes of the $e$-th thin plate element of ANCF, $\chi(\mathbf{r})$ is the TDF value defined in Eq. (30) at point $\mathbf{r}$ and $H(\chi)$ is the Heaviside function.

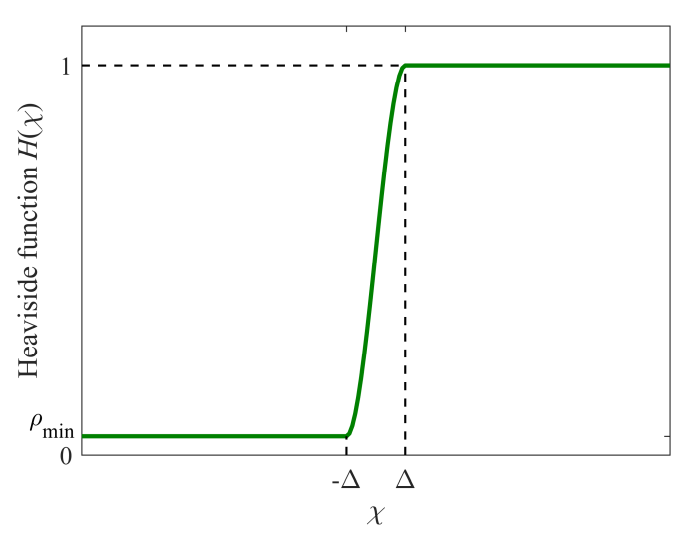

(a)

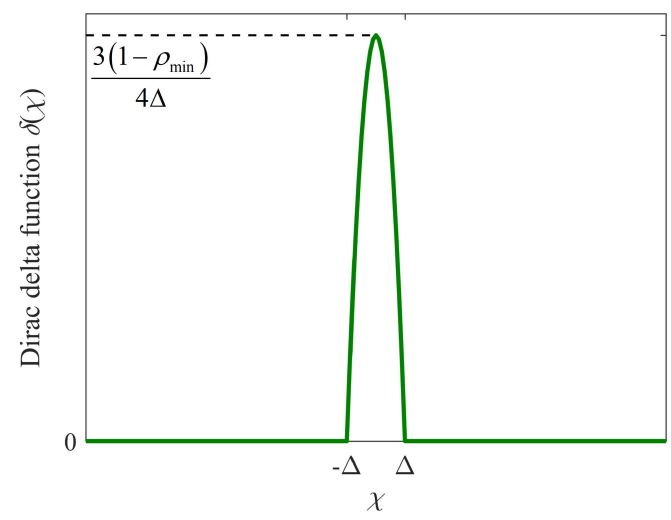

(b)

Fig. 5. Regularized Heaviside function and Dirac delta function. (a) Regularized Heaviside function; (b) regularized Dirac delta function.

For the purpose of numerical implementation, the Heaviside function $H(\chi)$ in Eq. (31) is regularized in the following way [27, 38, 41-43], as shown in Fig. 5(a)

$$
H(\chi)=\left\{\begin{array}{cc}
\frac{3\left(1-\rho_{\min }\right)}{4}\left(\frac{\chi}{\Delta}-\frac{\chi^{3}}{3 \Delta^{3}}\right)+\frac{1+\rho_{\min }}{2}, & (-\Delta \leq \chi \leq \Delta) \\
\rho_{\min }, & (\chi<-\Delta)
\end{array}\right.
$$


where $\Delta$ describes the width of the numerical approximation for $H(\chi) . \rho_{\min }$ is a very small positive number that denotes the volume fraction of weak material in the ersatz material model to ensure the nonsingularity of the stiffness matrix. In this study, $\rho_{\min }$ is given as $1 \times 10^{-6}$. The derivative function of $H(\chi)$ is called the Dirac delta function $\delta(\chi)$, as shown in Fig. 5(b), which can also be regularized according to Eq. (32) as

$$
\delta(\chi)=\left\{\begin{array}{cc}
\frac{3\left(1-\rho_{\min }\right)}{4 \Delta}\left(1-\frac{\chi^{2}}{\Delta^{2}}\right), & (|\chi| \leq \Delta) \\
0, & (|\chi|>\Delta)
\end{array}\right.
$$

For the topology optimization for eigenfrequencies of a structure, it is necessary to pay attention to the possibility of localized modes in the low-density areas [20]. In the present study, a fixed ANCF mesh is used and the ersatz material model in Eqs. (31) and (32) is employed to describe the properties of the finite elements of ANCF. Therefore, the localized modes may occur in the areas that have the minimum volume fraction $\rho \min$ or are close to the minimum volume fraction. This is due to the fact that these areas are so flexible relative to the mass that they may control the lowest eigenfrequencies of the entire structure. To remove the localized modes, Pedersen [20] proposed a combined method by introducing a new penalization scheme for the stiffness and ignoring some nodes when solving the eigenvalue problem in Eq. (22).

In this study, according to the work by Pedersen [20] and by Pedersen and Pedersen [44], the penalization schemes of the mass and stiffness can be respectively expressed as

$$
\begin{aligned}
& g_{1}\left(\rho_{e}\right)=\left\{\begin{array}{l}
\rho_{e}, \quad 0.01<\rho_{e} \leq 1 \\
\rho_{e}^{3}, \quad 0<\rho_{\min } \leq \rho_{e} \leq 0.01
\end{array}\right. \\
& g_{2}\left(\rho_{e}\right)=m_{4}(1-s) s+s^{2},
\end{aligned}
$$

where $s=m_{1}-\sqrt{m_{2}+m_{3} \rho_{e}}$ with $0<\rho_{\min } \leq \rho_{e} \leq 1$, and the four parameters $m_{1}, m_{2}$, $m_{3}$, and $m_{4}$ are defined as

$$
m_{1}=\frac{\kappa_{1}-1}{\kappa_{0}+\kappa_{1}-2}, m_{2}=m_{1}^{2}, m_{3}=\frac{\kappa_{0}-\kappa_{1}}{\kappa_{0}+\kappa_{1}-2}, m_{4}=\frac{2 \kappa_{0}\left(1-\kappa_{1}\right)}{\kappa_{0}-\kappa_{1}} .
$$

Here, $\kappa_{0}=g_{2}^{\prime}(0)$ and $\kappa_{1}=g_{2}^{\prime}(1)$ denote the two end slopes of the penalization function $g_{2}\left(\rho_{e}\right)$ in Eq. (34) with the expression of $g_{2}^{\prime}\left(\rho_{e}\right)$ as follows

$$
g_{2}^{\prime}\left(\rho_{e}\right)=\frac{\partial g_{2}\left(\rho_{e}\right)}{\partial \rho_{e}}=\frac{-\kappa_{1} s+\kappa_{0}\left(1-s+\kappa_{1}(2 s-1)\right)}{1+\kappa_{1}(s-1)+s\left(\kappa_{0}-2\right)}
$$

According to the work by Pedersen and Pedersen [44], the choice of $\kappa_{0}$ and $\kappa_{1}$ should satisfy $0 \leq \kappa_{0}<1, \kappa_{1}>1, \kappa_{0}+\kappa_{1}>2$. In order to remove the possibility of localized modes, the two parameters are chosen as $\kappa_{0}=0.1$ and $\kappa_{1}=2$, respectively.

As the first step, from Eq. (34), the mass matrix, the vector of generalized elastic forces, the vector of generalized centrifugal forces in Eq. (18) and the tangent stiffness matrix in Eq. (21) of the system can then be reformulated as follows 


$$
\left\{\begin{aligned}
& \mathbf{M}(\mathbf{b})=\sum_{e=1}^{N E 1} g_{1}\left(\rho_{e}\right) \mathbf{B}_{e}^{\mathrm{T}} \mathbf{M}_{e} \mathbf{B}_{e}+\sum_{e=1}^{N E 2} \mathbf{B}_{e}^{\mathrm{T}} \mathbf{M}_{e}^{*} \mathbf{B}_{e}, \\
& \mathbf{F}(\mathbf{b}, \mathbf{q}(\mathbf{b}))=\sum_{e=1}^{N E 1} g_{2}\left(\rho_{e}\right) \mathbf{B}_{e}^{\mathrm{T}} \mathbf{F}_{e}+\sum_{e=1}^{N E 2} \mathbf{B}_{e}^{\mathrm{T}} \mathbf{F}_{e}, \\
& \mathbf{Q}(\mathbf{b}, \mathbf{q}(\mathbf{b}))=\sum_{e=1}^{N E 1} g_{1}\left(\rho_{e}\right) \mathbf{B}_{e}^{\mathrm{T}} \mathbf{Q}_{e}+\sum_{e=1}^{N E 2} \mathbf{B}_{e}^{\mathrm{T}} \mathbf{Q}_{e}^{*}, \\
& \mathbf{K}(\mathbf{b}, \mathbf{q}(\mathbf{b}))=\sum_{e=1}^{N E 1} g_{2}\left(\rho_{e}\right) \mathbf{B}_{e}^{\mathrm{T}} \frac{\partial \mathbf{F}_{e}}{\partial \mathbf{e}} \mathbf{B}_{e}+\sum_{e=1}^{N E 2} \mathbf{B}_{e}^{\mathrm{T}} \frac{\partial \mathbf{F}_{e}}{\partial \mathbf{e}} \mathbf{B}_{e} \\
&-\sum_{e=1}^{N E 1} g_{1}\left(\rho_{e}\right) \mathbf{B}_{e}^{\mathrm{T}} \frac{\partial \mathbf{Q}_{e}}{\partial \mathbf{e}} \mathbf{B}_{e}-\sum_{e=1}^{N E 2} \mathbf{B}_{e}^{\mathrm{T}} \frac{\partial \mathbf{Q}_{e}^{*}}{\partial \mathbf{e}} \mathbf{B}_{e},
\end{aligned}\right.
$$

where $\mathbf{b}$ is the vector of design variables. It is worth noting that $\mathbf{M}$ only depends on $\mathbf{b}$, while $\mathbf{F}, \mathbf{Q}$, and $\mathbf{K}$ are functions of both $\mathbf{b}$ and $\mathbf{q}$ which also depends on $\mathbf{b}$.

As the second step, in order to obtain the correct eigenmodes, some nodal degrees of freedom are ignored when solving the eigenvalue problem (22). The ignored nodes are surrounded by the finite elements that all have a volume fraction of less than 0.01 . The ignorance is implemented by setting the deflections of these nodes to zeros in each iteration step of the subspace iteration method [36, 37], and removing them from the convergence condition [20].

\subsection{Optimization formulations for eigenfrequencies}

In this study, two objectives are considered for the optimization of the rotating thin plate, i.e., the maximization of the first eigenfrequency and the maximization of the gap between two eigenfrequencies of order $k$ and $k+1(k \geq 1)$.

The optimization formulation for maximizing the first eigenfrequency of the rotating thin plate can be mathematically expressed as the following bound formulation

$$
\begin{array}{cl}
\underset{\mathbf{b} \in \mathbb{R}^{N_{b}}}{\operatorname{maximize}} & f=C_{1} \\
\text { subject to } & \omega_{i}^{2} \geq C_{1} \quad\left(i=1,2, \cdots, N_{u}\right) \\
& \left(\mathbf{K}-\omega_{n}^{2} \mathbf{M}\right) \mathbf{Z}_{n}=\mathbf{0} \quad\left(n=1,2, \cdots, N_{d}\right) \\
& V(\mathbf{b})-V_{\text {spec }}=0 \\
& \mathbf{b} \in \mathcal{U}_{\mathbf{b}}
\end{array}
$$

where $\mathbf{b}=\left[\begin{array}{llll}\mathbf{b}_{1}^{\mathrm{T}} & \mathbf{b}_{2}^{\mathrm{T}} & \cdots & \mathbf{b}_{N_{c}}^{\mathrm{T}}\end{array}\right]^{\mathrm{T}}$ is the vector of $N_{b}$ design variables with $\mathbf{b}_{j}$ denoting the vector of design variables of the $j$-th component. Here, $N_{c}$ is the number of components, $N_{b}$ is the number of design variables and obviously, $N_{b}=7 N_{c}$ holds. The number $N_{u}\left(N_{u} \leq N_{d}\right)$ is chosen to be sufficiently large to ensure that all the eigenvalues higher than or equal to the first order are greater than $C_{1}$. Among the constraints, the second one is the eigenvalue problem with $N_{d}$ denoting the number of degrees of freedom for the system. The third constraint is the equality volume constraint, where $V(\mathbf{b})$ is the volume ratio of the rotating thin plate and $V_{\text {spec }}$ is a specified volume fraction. $\mathcal{U}_{\mathbf{b}}$ is the design space that belongs to. 
Similarly, the optimization formulation for maximizing the gap between two consecutive eigenfrequencies of order $k$ and $k+1$ of the rotating thin plate can be mathematically expressed as the following double bound formulation

$$
\begin{array}{cl}
\underset{\mathbf{b} \in \mathbb{R}^{N_{b}}}{\operatorname{maximize}} & f=C_{1}-C_{2} \\
\text { subject to } & \omega_{k+i}^{2} \geq C_{1} \quad\left(i=1,2, \cdots, N_{u}\right) \\
& \omega_{k+1-j}^{2} \leq C_{2} \quad\left(j=1,2, \cdots, N_{l}\right) \\
& \left(\mathbf{K}-\omega_{n}^{2} \mathbf{M}\right) \mathbf{Z}_{n}=\mathbf{0} \quad\left(n=1,2, \cdots, N_{d}\right) \\
& V(\mathbf{b})-V_{\text {spec }}=0 \\
& \mathbf{b} \in \mathcal{U}_{\mathbf{b}}
\end{array}
$$

where the numbers $N_{u}$ and $N_{l}$ are chosen to be large enough to make sure that all the eigenvalues of the order higher than or equal to $k+1$ are greater than $C_{1}$ and that all the eigenvalues of the order lower than or equal to $k$ stay under $C_{2}$.

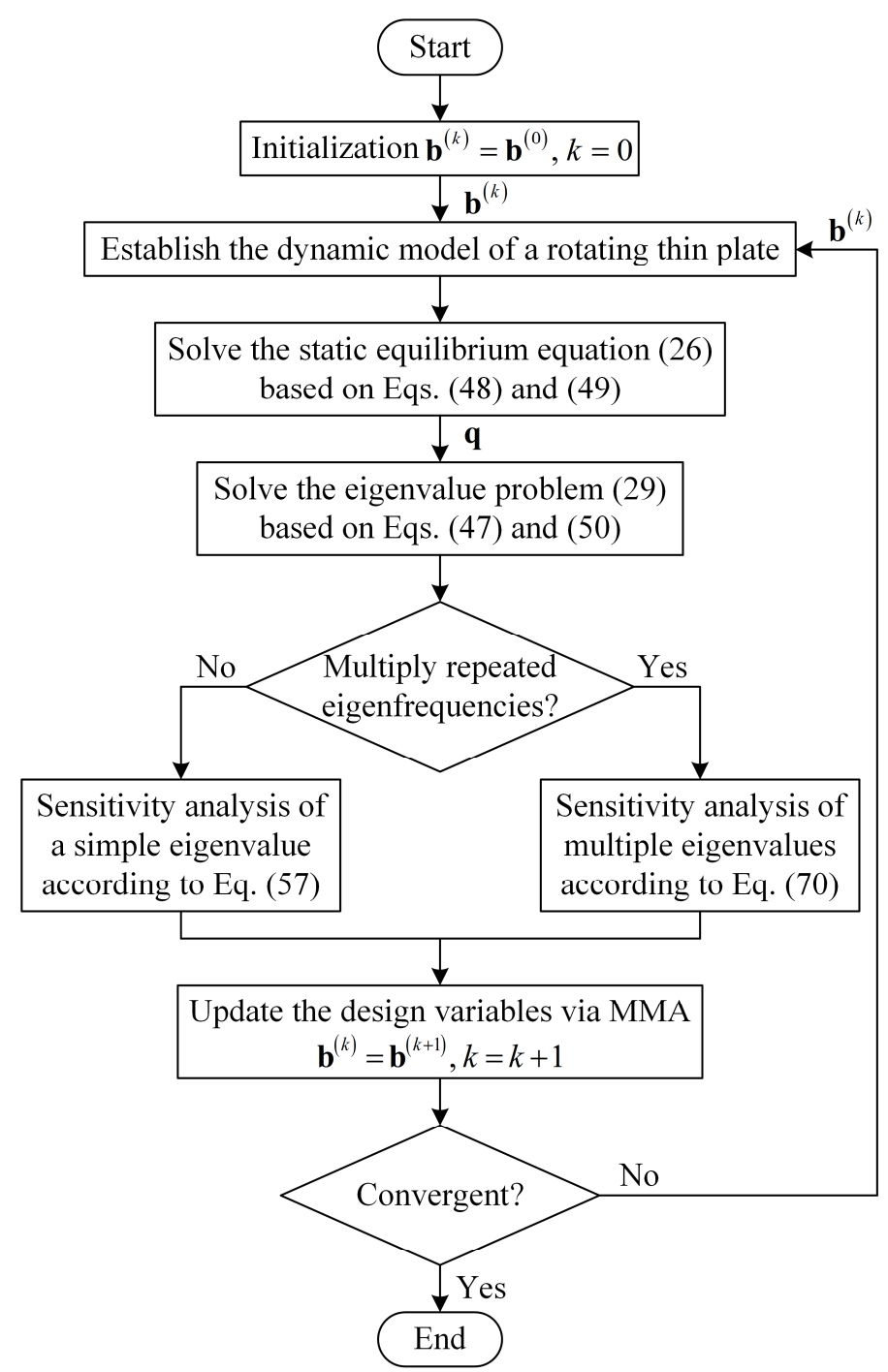

Fig. 6. The flowchart of the topology optimization for the eigenfrequencies of a rotating thin plate.

It can be seen that the optimization formulations in Eqs. (38) and (39) are two 
kinds of bound formulations by introducing variables $C_{1}$ and $C_{2}$ and moving the objective to the constraints. The merit of the bound formulation is that they can properly deal with the optimization cases with mode switching and multiply repeated eigenfrequencies and avoid the corresponding nonrobust convergence [19]. To solve the optimization problems formulated in Eqs. (38) and (39), it is natural to use the method of moving asymptotes (MMA) [45], which requires the sensitivities of the eigenfrequencies. Fig. 6 gives the flowchart of the topology optimization for the eigenfrequencies of a rotating thin plate.

\section{Sensitivity analysis}

\subsection{Sensitivities of a simple eigenfrequency}

In this subsection, the sensitivities of a simple eigenfrequency are derived. For a simple eigenpair $\left(\omega^{2}, \mathbf{Z}\right)$, in order to find the sensitivity of the eigenvalue $\omega^{2}$, one can directly take the derivative of the following eigenvalue problem in Eq. (40) with respective to a design variable

$$
\mathbf{Z}^{\mathrm{T}}\left(\mathbf{K}(\mathbf{b}, \mathbf{q}(\mathbf{b}))-\omega^{2} \mathbf{M}(\mathbf{b}, \mathbf{q}(\mathbf{b}))\right) \mathbf{Z}=\mathbf{0} .
$$

However, the result will contain the derivative of the vector of generalized coordinates $\mathbf{q}$ with respect to the design variable and needs time-consuming computations. Therefore, it is beneficial to use the adjoint method [46] to perform the sensitivity analysis. For this purpose, the eigenvalue problem in Eq. (40) is then augmented by the product of Eq. (20) and a vector of multipliers $\lambda$ as follows

$$
\mathbf{Z}^{\mathrm{T}}\left(\mathbf{K}(\mathbf{b}, \mathbf{q}(\mathbf{b}))-\omega^{2} \mathbf{M}(\mathbf{b}, \mathbf{q}(\mathbf{b}))\right) \mathbf{Z}+\lambda^{\mathrm{T}}(\mathbf{F}(\mathbf{b}, \mathbf{q}(\mathbf{b}))-\mathbf{Q}(\mathbf{b}, \mathbf{q}(\mathbf{b})))=\mathbf{0} .
$$

Taking the derivative of Eq. (41) with respect to an arbitrary design variable $b$ yields

$$
\begin{aligned}
\frac{\partial \omega^{2}}{\partial b} & =2 \mathbf{Z}^{\mathrm{T}}\left(\mathbf{K}-\omega^{2} \mathbf{M}\right) \frac{\partial \mathbf{Z}}{\partial b}+\left(\mathbf{Z}^{\mathrm{T}} \frac{\partial(\mathbf{K} \mathbf{Z})}{\partial \mathbf{q}}+\lambda^{\mathrm{T}} \mathbf{K}\right) \frac{\partial \mathbf{q}}{\partial b} \\
& +\mathbf{Z}^{\mathrm{T}}\left(\frac{\partial \mathbf{K}}{\partial b}-\omega^{2} \frac{\partial \mathbf{M}}{\partial b}\right) \mathbf{Z}+\boldsymbol{\lambda}^{\mathrm{T}}\left(\frac{\partial \mathbf{F}}{\partial b}-\frac{\partial \mathbf{Q}}{\partial b}\right) .
\end{aligned}
$$

It is assumed that the eigenvector $\mathbf{Z}$ is regularized so that $\mathbf{Z}^{\mathrm{T}} \mathbf{M Z}=1$. According to Eq. (40), the factor on the sensitivity of eigenvector $\partial \mathbf{Z} / \partial b$ in Eq. (42) is zero. To eliminate the term $\partial \mathbf{q} / \partial b$ in Eq. (42), the vector of multipliers $\lambda$ is determined by

$$
\mathbf{K}^{\mathrm{T}} \boldsymbol{\lambda}=-\left(\frac{\partial(\mathbf{K Z})}{\partial \mathbf{q}}\right)^{\mathrm{T}} \mathbf{Z} .
$$

It is worth noting that in the computation of $\partial(\mathbf{K Z}) / \partial \mathbf{q}$, the eigenvector $\mathbf{Z}$ is regarded as a constant vector. Now, with the definition of $\lambda$ in Eq. (43), the sensitivity of $\omega^{2}$ in Eq. (42) can be rewritten as 


$$
\frac{\partial \omega^{2}}{\partial b}=\mathbf{Z}^{\mathrm{T}}\left(\frac{\partial \mathbf{K}}{\partial b}-\omega^{2} \frac{\partial \mathbf{M}}{\partial b}\right) \mathbf{Z}+\lambda^{\mathrm{T}}\left(\frac{\partial \mathbf{F}}{\partial b}-\frac{\partial \mathbf{Q}}{\partial b}\right) .
$$

According to Eq. (37), the derivatives of $\mathbf{K}, \mathbf{M}, \mathbf{F}$, and $\mathbf{Q}$ with respect to an arbitrary design variable $b$ in Eq. (44) can be expressed as

$$
\left\{\begin{array}{l}
\frac{\partial \mathbf{K}}{\partial b}=\frac{1}{4} \sum_{e=1}^{N E 1} \sum_{i=1}^{4} \frac{\partial H\left(\chi\left(\mathbf{r}_{i}^{e}\right)\right)}{\partial b} \cdot\left(\frac{\partial g_{2}}{\partial \rho_{e}} \mathbf{B}_{e}^{\mathrm{T}} \frac{\partial \mathbf{F}_{e}}{\partial \mathbf{e}} \mathbf{B}_{e}-\frac{\partial g_{1}}{\partial \rho_{e}} \mathbf{B}_{e}^{\mathrm{T}} \frac{\partial \mathbf{Q}_{e}}{\partial \mathbf{e}} \mathbf{B}_{e}\right) \\
\frac{\partial \mathbf{M}}{\partial b}=\frac{1}{4} \sum_{e=1}^{N E 1} \sum_{i=1}^{4} \frac{\partial H\left(\chi\left(\mathbf{r}_{i}^{e}\right)\right)}{\partial b} \cdot \frac{\partial g_{1}}{\partial \rho_{e}} \mathbf{B}_{e}^{\mathrm{T}} \mathbf{M}_{e} \mathbf{B}_{e} \\
\frac{\partial \mathbf{F}}{\partial b}=\frac{1}{4} \sum_{e=1}^{N E 1} \sum_{i=1}^{4} \frac{\partial H\left(\chi\left(\mathbf{r}_{i}^{e}\right)\right)}{\partial b} \cdot \frac{\partial g_{2}}{\partial \rho_{e}} \mathbf{B}_{e}^{\mathrm{T}} \mathbf{F}_{e} \\
\frac{\partial \mathbf{Q}}{\partial b}=\frac{1}{4} \sum_{e=1}^{N E 1} \sum_{i=1}^{4} \frac{\partial H\left(\chi\left(\mathbf{r}_{i}^{e}\right)\right)}{\partial b} \cdot \frac{\partial g_{1}}{\partial \rho_{e}} \mathbf{B}_{e}^{\mathrm{T}} \mathbf{Q}_{e}
\end{array}\right.
$$

where $\partial H(\chi) / \partial b$ can be computed by $\partial H(\chi) / \partial b=\delta_{j}(\chi)\left(\partial \chi_{j} / \partial b\right)[25,27,47]$ with $\delta_{j}(\chi)=\min \left(\delta\left(\chi_{j}\right), \delta(\chi)\right)$, which indicates that $\delta_{j}(\chi(\mathbf{r})) \neq 0$ only holds for the points $\mathbf{r} \in \partial \Omega_{j} \cap \partial \Omega$. $\partial \chi_{j} / \partial b$ can be directly obtained from Eqs. (26), (27) and (28) due to the explicit description and will not be presented in this study. Zhang et al. [27] pointed out that $\partial H(\chi) / \partial b$ can also be computed by using the finite difference quotient of $\chi$, i.e., $\Delta H(\chi) / \Delta b$.

\subsection{Sensitivities of multiply repeated eigenfrequencies}

For the topology optimization for eigenfrequencies, it is important to pay attention to the possibility of multiply repeated eigenfrequencies for the sensitivity analysis [19, 31]. For simplicity, a pair of repeated eigenfrequencies with two corresponding eigenvectors $\left(\omega^{2}, \mathbf{Z}_{1}, \mathbf{Z}_{2}\right)$ is considered, but the derivative can be straightforwardly extended to a higher number of multiplicity. It is assumed that the two eigenvectors $\mathbf{Z}_{1}$ and $\mathbf{Z}_{2}$ are normalized with respect to the mass matrix and are orthogonal with each other as $\mathbf{Z}_{1}^{\mathrm{T}} \mathbf{M} \mathbf{Z}_{1}=1, \mathbf{Z}_{2}^{\mathrm{T}} \mathbf{M} \mathbf{Z}_{2}=1, \mathbf{Z}_{1}^{\mathrm{T}} \mathbf{M} \mathbf{Z}_{2}=0$.

Jensen and Pedersen [19] pointed out that the sensitivities of the multiply repeated eigenfrequencies depend not only on the changes of the design variables, but also on the choices of the eigenvector. This is due to the fact that for a pair of repeated eigenfrequencies, any linear combination of the two eigenvectors $\mathbf{Z}_{1}$ and $\mathbf{Z}_{2}$ is also its eigenvector as follows

$$
\mathbf{Z}=a_{1} \mathbf{Z}_{1}+a_{2} \mathbf{Z}_{2}
$$

where $a_{1}, a_{2} \in[-1,1]$ and $a_{1}^{2}+a_{2}^{2}=1$ so that $\mathbf{Z}^{\mathrm{T}} \mathbf{M Z}=1$.

Then, by substituting Eq. (46) into Eq. (44), one has 


$$
\frac{\partial \omega^{2}}{\partial b}=a_{1}^{2} A_{11}+a_{2}^{2} A_{22}+2 a_{1} a_{2} A_{12}
$$

where $A_{11}, A_{12}$, and $A_{22}$ can be expressed as

$$
A_{\alpha \beta}=\mathbf{Z}_{\alpha}^{\mathrm{T}}\left(\frac{\partial \mathbf{K}}{\partial b}-\omega^{2} \frac{\partial \mathbf{M}}{\partial b}\right) \mathbf{Z}_{\beta}+\lambda_{\alpha \beta}^{\mathrm{T}}\left(\frac{\partial \mathbf{F}}{\partial b}-\frac{\partial \mathbf{Q}}{\partial b}\right)
$$

with $\alpha, \beta=1,2$ and $\boldsymbol{\lambda}_{\alpha \beta}$ defined by $\mathbf{K}^{\mathrm{T}} \boldsymbol{\lambda}_{\alpha \beta}=-\left(\partial\left(\mathbf{K} \mathbf{Z}_{\beta}\right) / \partial \mathbf{q}\right)^{\mathrm{T}} \mathbf{Z}_{\alpha}$.

In order to find the extreme values of $\partial \omega^{2} / \partial b$ subject to $a_{1}^{2}+a_{2}^{2}=1$, Eq. (47) is augmented as $\mathcal{L}=a_{1}^{2} A_{11}+a_{2}^{2} A_{22}+2 a_{1} a_{2} A_{12}+\lambda\left(1-a_{1}^{2}-a_{2}^{2}\right)$. Then, differentiating $\mathcal{L}$ with respect to $a_{1}, a_{2}$, and $\lambda$ and setting these equations to zeros will lead to

$$
\left[\begin{array}{cc}
A_{11}-\lambda & A_{12} \\
A_{12} & A_{22}-\lambda
\end{array}\right]\left[\begin{array}{l}
a_{1} \\
a_{2}
\end{array}\right]=\left[\begin{array}{l}
0 \\
0
\end{array}\right]
$$

which is subject to $a_{1}^{2}+a_{2}^{2}=1$. Solving Eq. (49) is equivalent to finding the eigenvalues and unit eigenvectors of the matrix $\mathbf{A}=\left[\begin{array}{ll}A_{11} & A_{12} \\ A_{12} & A_{22}\end{array}\right]$, i.e., $\left(\lambda_{1},\left[\begin{array}{l}a_{11} \\ a_{21}\end{array}\right]\right)$ and $\left(\lambda_{2},\left[\begin{array}{l}a_{12} \\ a_{22}\end{array}\right]\right)$

Now, the sensitivities of a pair of repeated eigenfrequencies in Eq. (47) can be expressed as

$$
\frac{\partial \omega^{2}}{\partial b}= \begin{cases}\lambda_{1} & \text { with eigenvector } \tilde{\mathbf{Z}}_{1}=a_{11} \mathbf{Z}_{1}+a_{21} \mathbf{Z}_{2} \\ \lambda_{2} & \text { with eigenvector } \tilde{\mathbf{Z}}_{2}=a_{12} \mathbf{Z}_{1}+a_{22} \mathbf{Z}_{2}\end{cases}
$$

It is worth mentioning that for different design variables, the eigenvectors $\tilde{\mathbf{Z}}_{1}$ and $\tilde{\mathbf{Z}}_{2}$ are different due to the changes of $A_{\alpha \beta}$ in Eq. (48). This implies that the sensitivities in Eq. (50) are given for two specific directions $\tilde{\mathbf{Z}}_{1}$ and $\tilde{\mathbf{Z}}_{2}$ in the space spanned by $\mathbf{Z}_{1}$ and $\mathbf{Z}_{2}$.

\section{Numerical examples}

\subsection{Modal analysis to remove localized modes in low-density areas}

This is a classic example firstly studied by Pedersen [20] to illustrate how to remove the localized modes in the low-density areas when performing the topology optimization for eigenfrequencies of the plate. In this subsection, the example is used to show that the localized modes in the low-density areas can be removed for the rotating thin plate at different angular velocities. As shown in Fig. 7, the two equivalent cantilever thin plates both have the same solid areas, i.e., $1 \mathrm{~m} \times 0.2 \mathrm{~m}$. For the solid areas, Young's modulus E, Poison's ratio $v$, and mass density $\rho$ are $1.8 \times 10^{11} \mathrm{~Pa}, 0.06$, and 2300 
$\mathrm{kg} / \mathrm{m}^{3}$, respectively. For the case in Fig. 7(b), there are no low-density areas, while in Fig. 7(a), the low-density areas are symmetrically distributed on the upper and lower sides of the solid areas. In the low-density areas, the volume fraction $\rho_{e}$ is $1 \times 10^{-6}$. The thickness of the thin plate is $0.01 \mathrm{~m}$ for the two cases in Fig. 7.

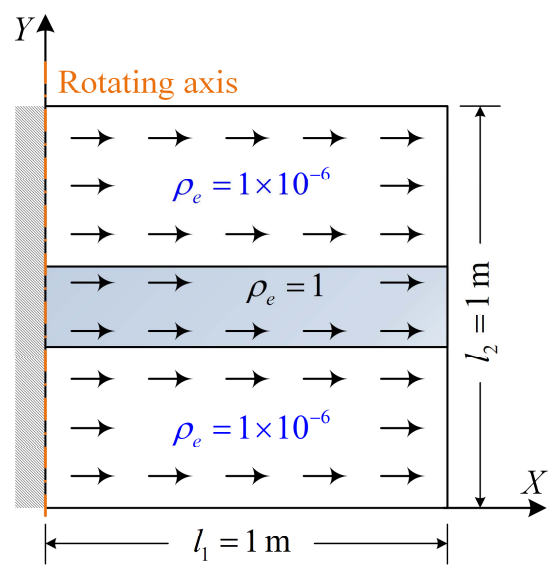

(a)



(b)

Fig. 7. The equivalent cantilever thin plate for the rotating thin plate in two cases with and without lowdensity areas. (a) Case $1: \beta=1$; (b) case $2: \beta=5$.

Table 1 presents the dimensionless eigenfrequencies of the first four orders of the rotating thin plate for the two cases in Fig. 7. The table shows that the two cases in Fig. 7 get a perfect agreement for the eigenfrequencies of the first three orders, and there is only a small difference between the two cases when $\gamma=4,8,9$, and 10 for the fourthorder eigenfrequency. This phenomenon indicates that the localized modes in the lowdensity areas in Fig. 7(a) can be well removed. The penalization schemes of the mass and stiffness in Section 4.2, therefore, are appropriate for the MMC-based topology optimization for eigenfrequencies of a rotating thin plate. Fig. 8 illustrates the first four mode shapes of the rotating thin plate when $\gamma=0$ and shows that the orders of the mode shapes also agree with each other for the two cases in Fig. 7.

Table 1 Dimensionless eigenfrequencies of the rotating thin plate for the two cases with and without low-density areas $(\mu=100, \sigma=0)$.

\begin{tabular}{|c|c|c|c|c|c|c|c|c|}
\hline \multirow{2}{*}{$\gamma$} & \multicolumn{4}{|c|}{ Case $1: \beta=1$} & \multicolumn{4}{|c|}{ Case $2: \beta=5$} \\
\hline & $\overline{\omega_{1}}$ & $\bar{\omega}_{2}$ & $\bar{\omega}_{3}$ & $\overline{\bar{\omega}_{4}}$ & $\overline{\bar{\omega}_{1}}$ & $\bar{\omega}_{2}$ & $\bar{\omega}_{3}$ & $\bar{\omega}_{4}$ \\
\hline 0 & 3.5115 & 22.0059 & 38.9899 & 61.6262 & 3.5115 & 22.0059 & 38.9899 & 61.6262 \\
\hline 1 & 3.6774 & 22.1525 & 39.0036 & 61.7706 & 3.6774 & 22.1525 & 39.0036 & 61.7706 \\
\hline 2 & 4.1335 & 22.5866 & 39.0447 & 62.2015 & 4.1335 & 22.5866 & 39.0447 & 62.2015 \\
\hline 3 & 4.7940 & 23.2923 & 39.1130 & 62.9124 & 4.7940 & 23.2923 & 39.1130 & 62.9124 \\
\hline 4 & 5.5822 & 24.2457 & 39.2085 & 63.8931 & 5.5822 & 24.2457 & 39.2085 & 63.8930 \\
\hline 5 & 6.4471 & 25.4188 & 39.3308 & 65.1299 & 6.4471 & 25.4188 & 39.3308 & 65.1299 \\
\hline 6 & 7.3581 & 26.7822 & 39.4797 & 66.6070 & 7.3581 & 26.7822 & 39.4797 & 66.6070 \\
\hline 7 & 8.2975 & 28.3075 & 39.6548 & 68.3071 & 8.2975 & 28.3075 & 39.6548 & 68.3071 \\
\hline 8 & 9.2548 & 29.9692 & 39.8558 & 69.2374 & 9.2548 & 29.9691 & 39.8558 & 69.2371 \\
\hline 9 & 10.2237 & 31.7445 & 40.0822 & 69.3812 & 10.2237 & 31.7445 & 40.0822 & 69.3809 \\
\hline 10 & 11.2003 & 33.6146 & 40.3336 & 69.5416 & 11.2003 & 33.6146 & 40.3336 & 69.5413 \\
\hline
\end{tabular}



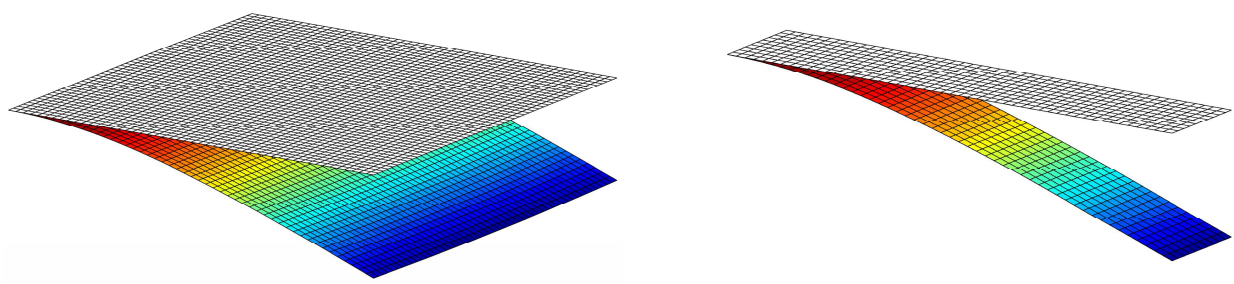

(a)
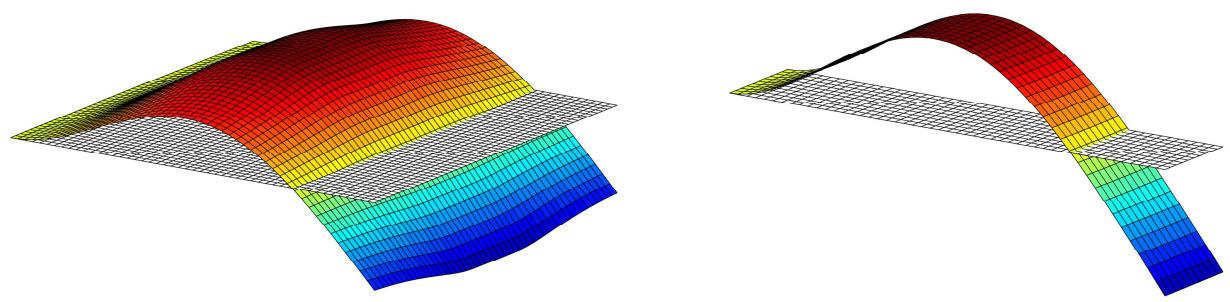

(b)
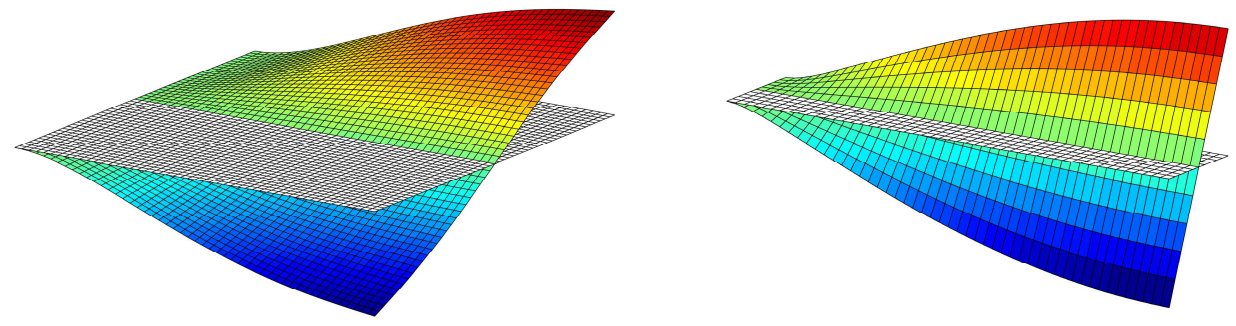

(c)
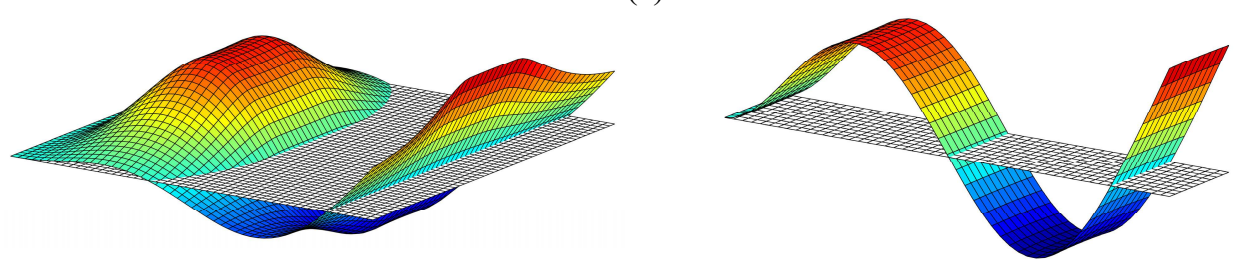

(d)

Fig. 8. The first four mode shapes of the rotating thin plate for the two cases with and without lowdensity areas $(\mu=100, \sigma=0, \gamma=0$ ). (a) First-order mode shape; (b) second-order mode shape; (c) third-order mode shape; $(d)$ fourth-order mode shape.

\subsection{Maximizing the first eigenfrequency of the rotating thin plate without nonstructural} mass

This subsection shows how to validate the proposed topology optimization approach for the first eigenfrequency of a rotating thin plate without nonstructural mass at the tip edge. The rotating thin plate of concern has a length of $l_{1}=1 \mathrm{~m}$ and a width of $l_{2}=1 \mathrm{~m}$, a thickness of $l_{3}=0.001 \mathrm{~m}$. Young's modulus $E$, Poison's ratio $v$, and mass density $\rho$ of the plate material are $7.1 \times 10^{10} \mathrm{~Pa}, 0.3$, and $2750 \mathrm{~kg} / \mathrm{m}^{3}$, respectively. The volume fraction $V_{\text {spec }}$ is set as 0.5 .

Theoretically speaking, to maximize the first eigenfrequency of such a rotating thin plate without nonstructural mass, all the masses will move to the left part of the plate or be totally removed. Fig. 9 illustrates that all the components have occupied the left half part of the rotating thin plate after convergence for the case of $\beta=1, \mu=1000$, $\sigma=0, \gamma=0$. In Fig. 9, each iteration step presents the contour plot (the left one in teal) and the component plot (the right one in red). It can be seen that only four components are used to describe the topology of the thin plate so that the number of the design variables 
is 28. Fig. 10 shows the iteration histories of the objective function and the volume ratio. From Fig. 10, it can be observed that the optimization process converges fast with no more than 200 iteration steps. After convergence, the equality volume constraint $(V=0.5)$ is satisfied. The converged objective function value is 464.8490 which is quite close to 461.4984 , i.e., the first eigenvalue $\omega_{1}^{2}$ of the thin plate with only a half part of materials on the left.
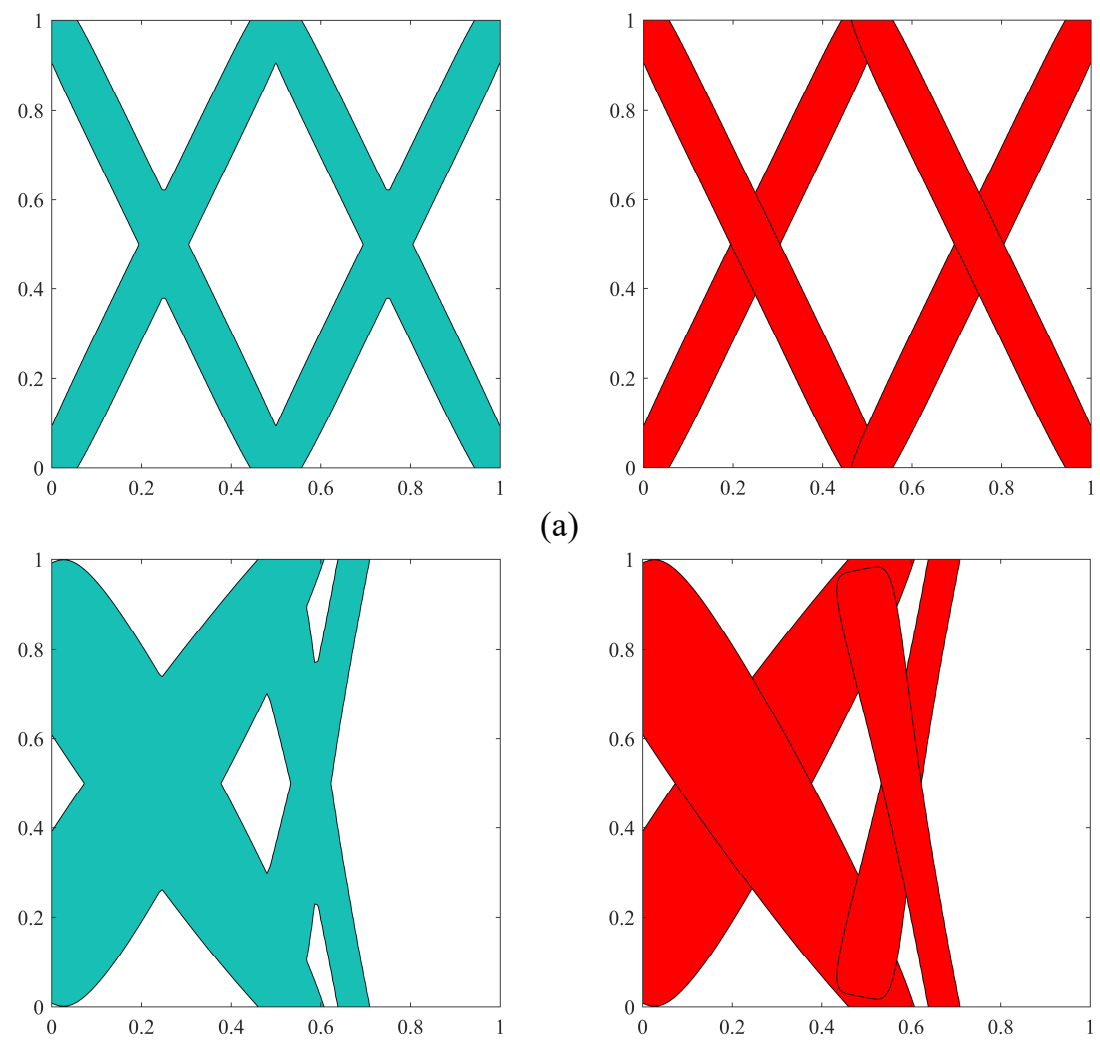

(a)
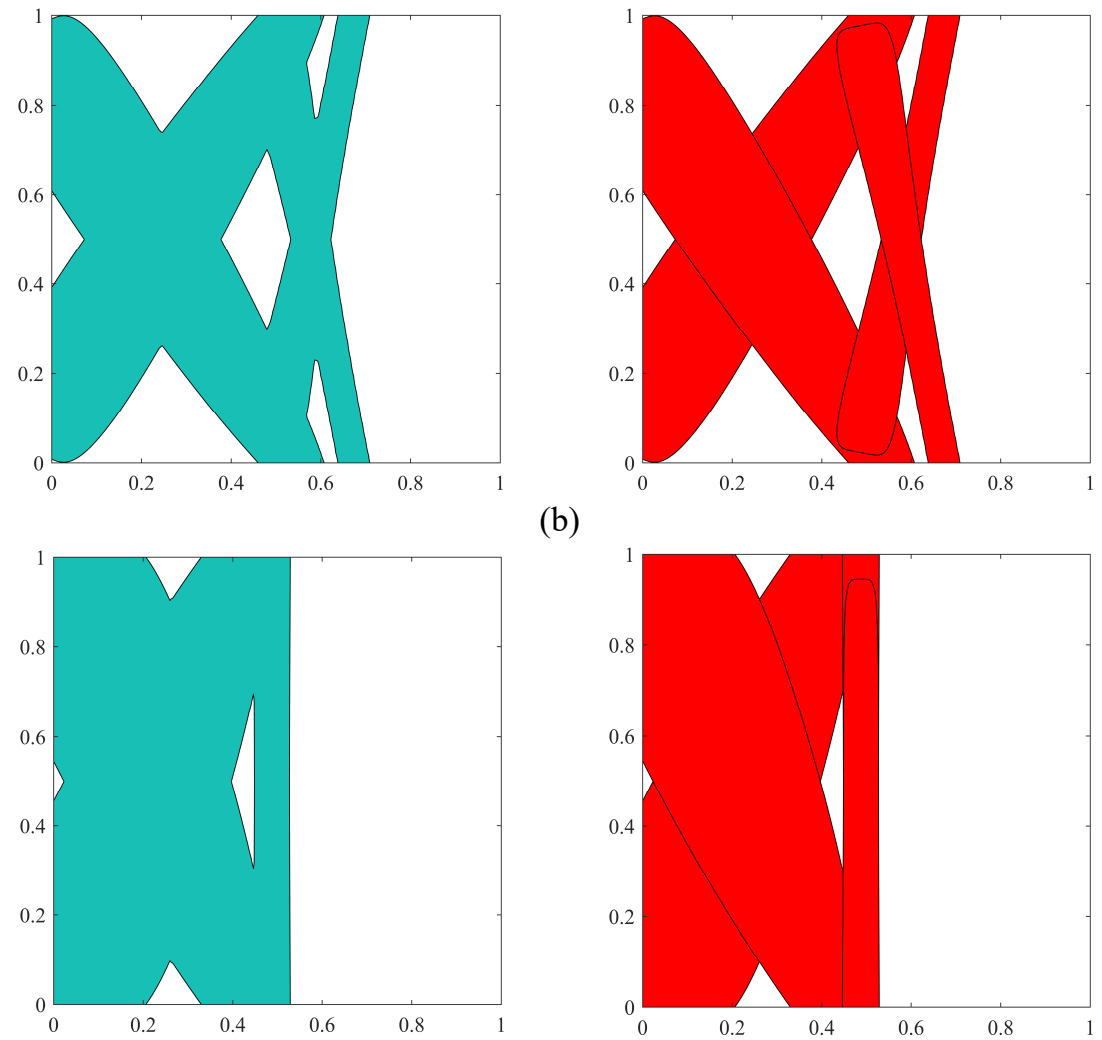

(b)

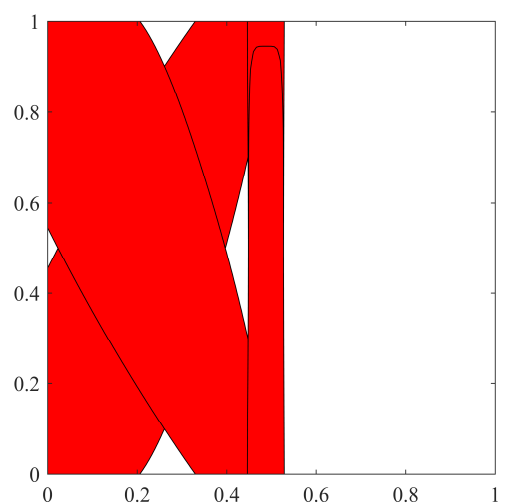

(c) 

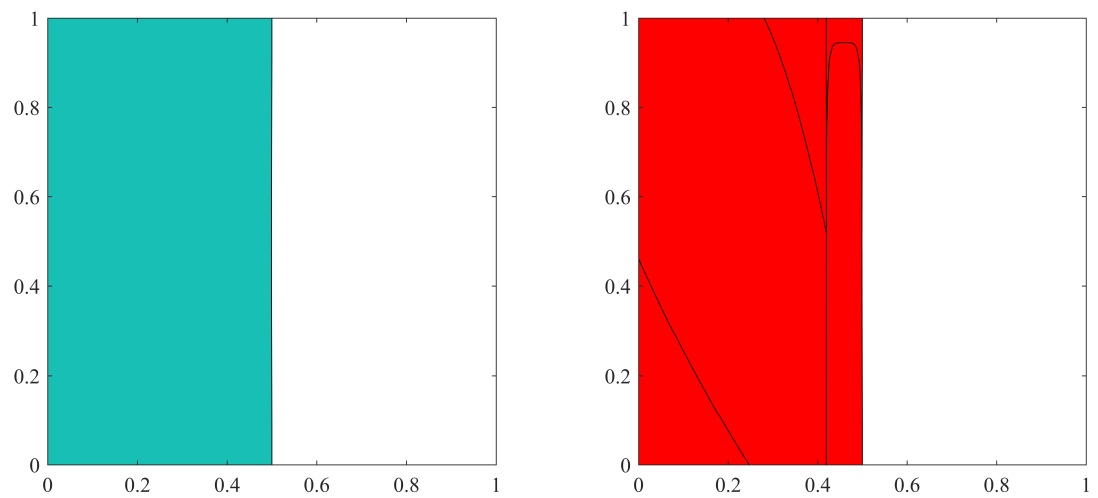

(d)

Fig. 9. Optimized results of the rotating thin plate without nonstructural mass $(\beta=1, \mu=1000, \sigma=0, \gamma=0)$ : contour plot (left) and component plot (right). (a) Iteration No. 1; (b) iteration No. 50; (c) iteration No. 100; (d) iteration No. 200.



Fig. 10. Iteration histories of the objective function and the volume ratio $(\beta=1, \mu=1000, \sigma=0, \gamma=0)$.

6.3. Maximizing the first eigenfrequency of the rotating thin plate with nonstructural mass

This subsection shows how to optimize the rotating thin plate with an initial topology in Fig. 11 for maximizing its first eigenfrequency when two different nonstructural masses are taken into consideration. In Fig. 11(a), the nonstructural mass occupies the whole tip edge of the rotating thin plate with a length of $l_{1}^{*}=0.05 l_{1}$ and a width of $l_{2}^{*}=l_{2}$. In Fig. 11(b), the nonstructural mass only occupies a part of the tip edge and is centered vertically with a length of $l_{1}^{*}=0.2 l_{1}$ and a width of $l_{2}^{*}=0.3 l_{2}$. For the two cases, the mass density of the nonstructural mass is equal to the mass density of the solid domain, i.e., $\rho^{*}=\rho$. In this example, the rotating thin plate has the same geometrical parameters and material parameters as those in Section 6.2. The radius of the rigid hub $R$ is 0 . The volume fraction $V_{\text {spec }}$ is set as 0.5 as well.

From Fig. 11, it can be seen that the same number and configuration of the components are placed in the design domain for the two cases. According to the symmetry of the rotating thin plate, only 30 components are employed to describe the topology of the top half part of the rotating thin plate. The bottom half part of the rotating thin plate, therefore, is mirror-symmetrical to the top half. As a result, the 
number of design variables is 210 .

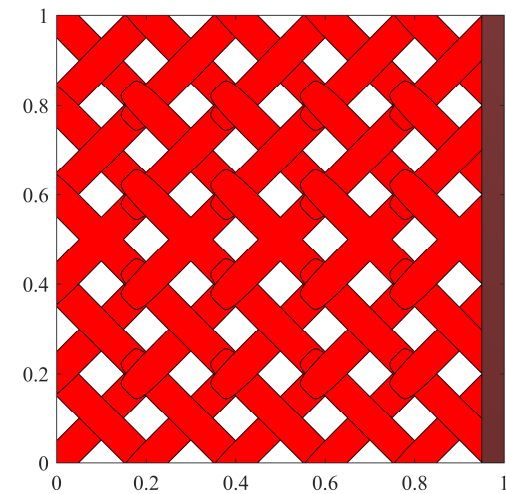

(a)

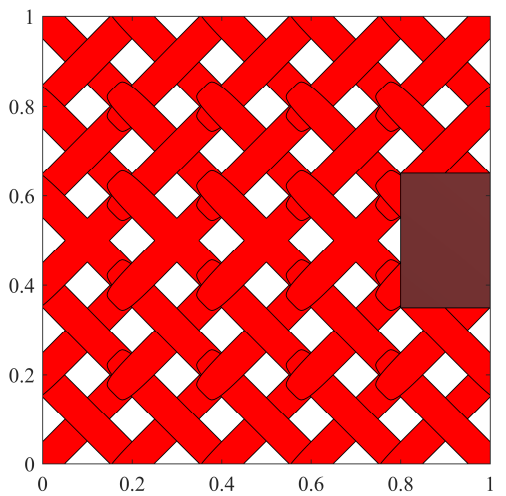

(b)

Fig. 11. Initial configurations of the components for the two cases with different nonstructural masses $(\alpha=1, \beta=1, \mu=1000, \sigma=0)$. (a) Case 1: $l_{1}^{*}=0.05 l_{1}, l_{2}^{*}=l_{2}$; (b) case $2: l_{1}^{*}=0.2 l_{1}, l_{2}^{*}=0.3 l_{2}$.

Table 2 Dimensionless first eigenfrequencies $\bar{\omega}_{1}$ of the unoptimized and optimized rotating thin plates in Fig. 12 and Fig. $14(\alpha=1, \beta=1, \mu=1000, \sigma=0)$.

\begin{tabular}{|c|c|c|c|c|c|c|}
\hline \multicolumn{2}{|c|}{$\bar{\omega}_{1}$} & $\gamma=0$ & $\gamma=1$ & $\gamma=2$ & $\gamma=4$ & $\gamma=8$ \\
\hline \multicolumn{2}{|c|}{$\begin{array}{l}\text { Unoptimized model } \\
\text { for Case } 1\end{array}$} & 2.3113 & 2.5567 & 3.1765 & 4.8953 & 8.7750 \\
\hline \multirow{5}{*}{$\begin{array}{l}\text { Optimized } \\
\text { models for } \\
\text { Case } 1\end{array}$} & Fig. 12(a) & 5.0930 & 5.2338 & 5.6299 & 6.9456 & 10.4458 \\
\hline & Fig. 12(b) & 5.0921 & 5.2393 & 5.6338 & 6.9453 & 10.4399 \\
\hline & Fig. 12(c) & 5.0899 & 5.2336 & 5.6366 & 6.9662 & 10.4720 \\
\hline & Fig. 12(d) & 4.9032 & 5.0771 & 5.5490 & 7.0010 & 10.5432 \\
\hline & Fig. 12(e) & 2.0895 & 2.9129 & 4.2752 & 6.6193 & 10.5729 \\
\hline \multicolumn{2}{|c|}{$\begin{array}{l}\text { Unoptimized model } \\
\text { for Case } 2\end{array}$} & 2.3169 & 2.5618 & 3.1808 & 4.8984 & 8.7772 \\
\hline \multirow{5}{*}{$\begin{array}{l}\text { Optimized } \\
\text { models for } \\
\text { Case } 2\end{array}$} & Fig. 14(a) & 5.5880 & 5.7098 & 6.0581 & 7.2599 & 10.6305 \\
\hline & Fig. 14(b) & 5.5875 & 5.7111 & 6.0628 & 7.2763 & 10.6567 \\
\hline & Fig. 14(c) & 5.5829 & 5.7076 & 6.0632 & 7.2803 & 10.6693 \\
\hline & Fig. 14(d) & 5.4946 & 5.6281 & 6.0063 & 7.2826 & 10.7221 \\
\hline & Fig. 14(e) & 1.7353 & 2.8783 & 4.5007 & 6.9291 & 10.7958 \\
\hline
\end{tabular}

Fig. 12 presents the optimized results for maximizing the first eigenfrequency of the rotating thin plate with the nonstructural mass in case 1 for five dimensionless angular velocities, i.e., $\gamma=0,1,2,4$, and 8 . Fig. 12 , shows that the optimized results have the same topology for $\gamma=0,1,2$, and 4 , but different shapes. For $\gamma=8$, the optimized topology is different from those in Fig. 12(a), (b), (c) and (d). Fig. 12 also gives the first eigenfrequencies of the optimized results. Compared with the unoptimized model as shown in Table 2, the first eigenfrequencies optimized increase from 2.3113, 2.5567, $3.1765,4.8953$ and 8.7750 to $5.0930,5.2393,5.6366,7.0010$ and 10.5729 , respectively for $\gamma=0,1,2,4$, and 8 . It is worth mentioning that the unoptimized model in Table 2 is the model with only a half part of materials evenly distributed in the design domain. Table 2 also gives the cross-check results for the optimized models in Fig. 12. The cross-check results indicate that all the five optimized models in Fig. 12 are successful as for each prescribed angular velocity, the first eigenfrequency of the optimized model is larger than the other four results. 

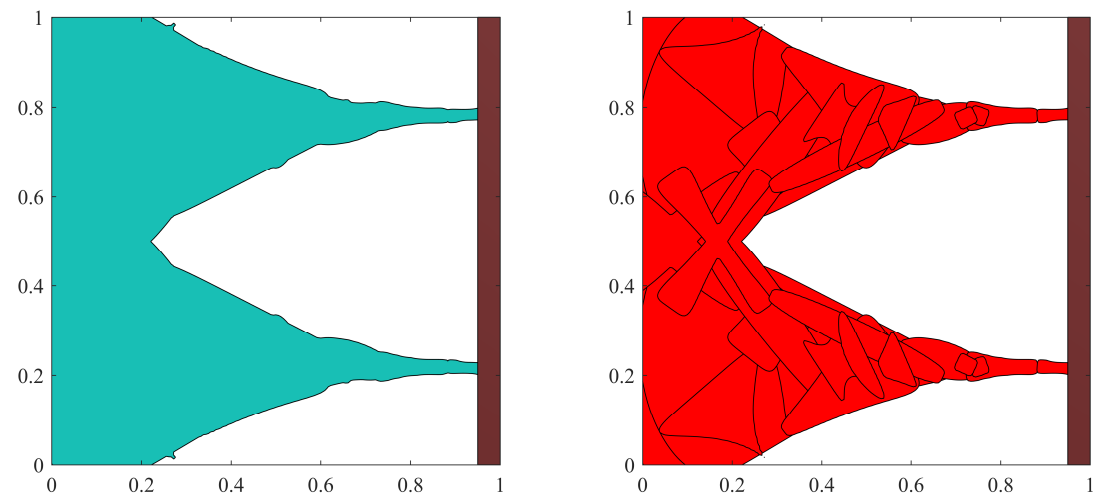

(a)
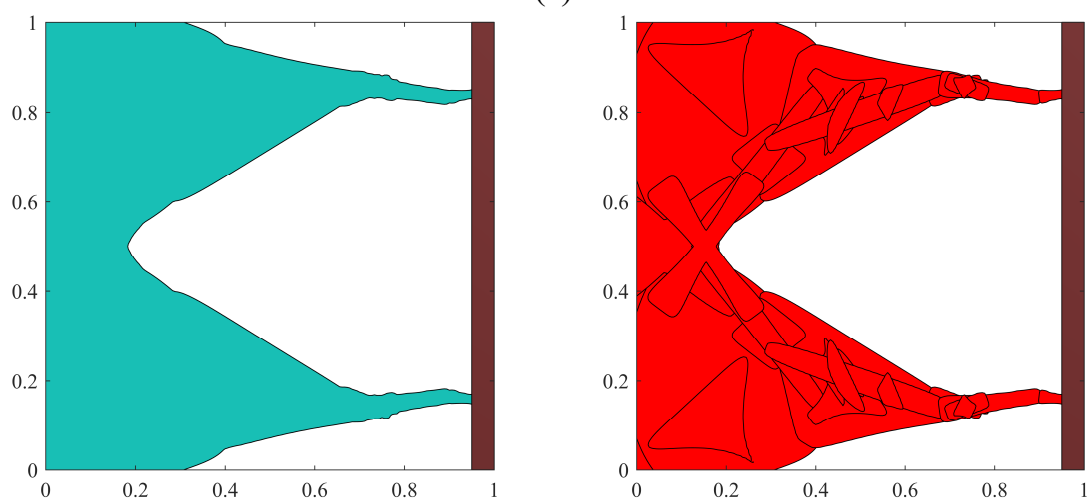

(b)
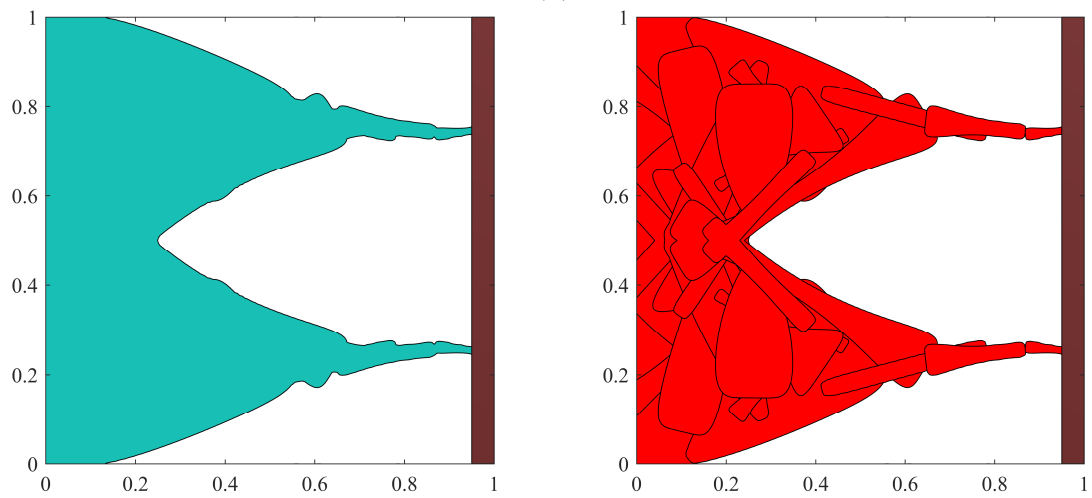

(c)
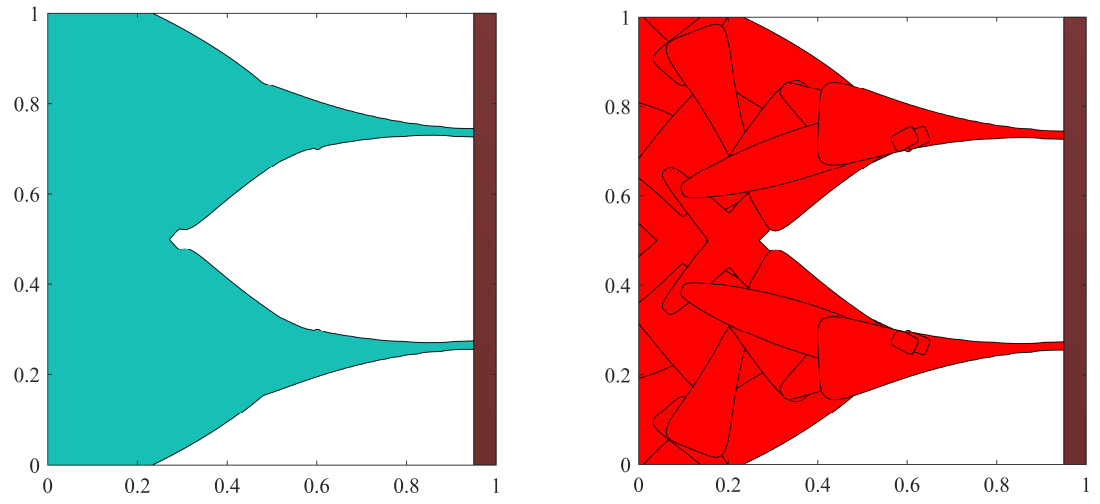

(d) 

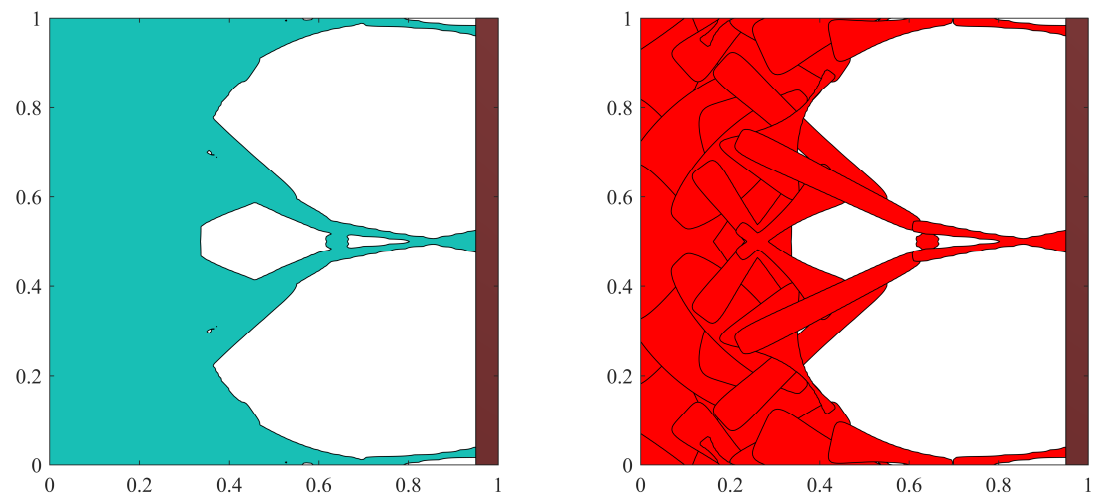

(e)

Fig. 12. Optimized results for maximizing the first eigenfrequency of the rotating thin plate with nonstructural mass: Case $1(\alpha=1, \beta=1, \mu=1000, \sigma=0)$. (a) $\gamma=0, \bar{\omega}_{1}=5.0930$; (b) $\gamma=1, \bar{\omega}_{1}=5.2393$; (c) $\gamma=2, \quad \bar{\omega}_{1}=5.6366 ;$ (d) $\gamma=4, \bar{\omega}_{1}=7.0010 ;$ (e) $\gamma=8, \bar{\omega}_{1}=10.5729$.

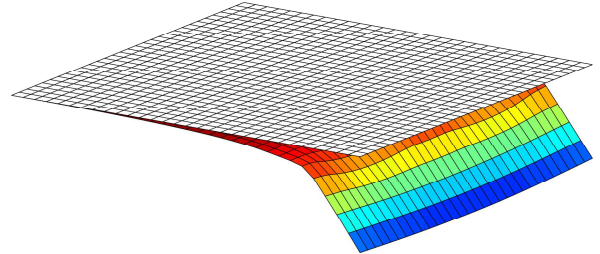

(a)

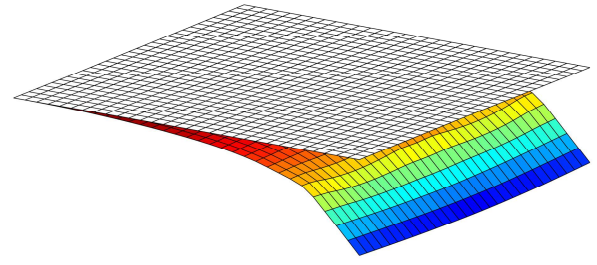

(c)

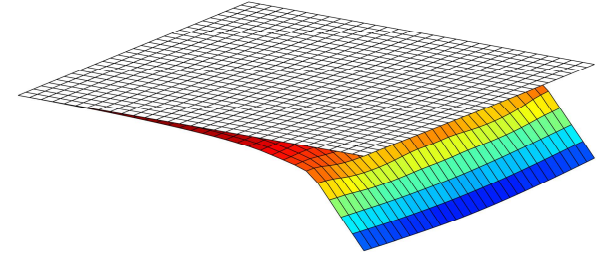

(b)

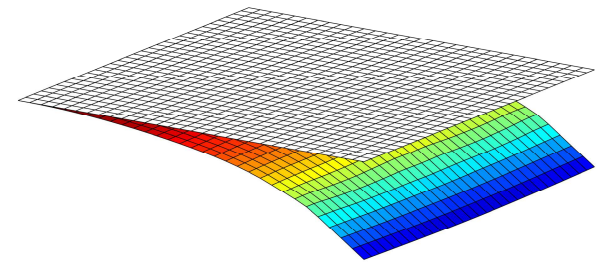

(d)

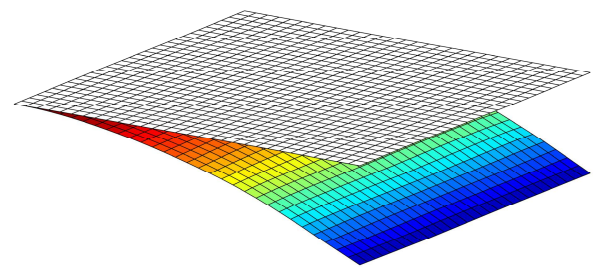

(e)

Fig. 13. First-order mode shapes of the optimized model in Fig. 12(e) at different angular velocities. (a) $\gamma=0, \quad \bar{\omega}_{1}=2.0895$; (b) $\gamma=1, \bar{\omega}_{1}=2.9129$; (c) $\gamma=2, \bar{\omega}_{1}=4.2752$; (d) $\gamma=4, \bar{\omega}_{1}=6.6193$; (e) $\gamma=8$, $\bar{\omega}_{1}=10.5729$.

From Fig. 12 and Table 2, it can also be seen that when the angular velocity is small $(\gamma \leq 4)$, the optimized topologies are the same and the first eigenfrequencies of the optimized models are quite close to each other for a prescribed angular velocity. When the angular velocity is large $(\gamma=8)$, however, the optimized model in Fig. 12(e) shows a different dynamic behavior from the other four models in Fig. 12(a), (b), (c) and (d). According to Table 2, the first eigenfrequencies of the optimized model in Fig. 12(e) are quite smaller than those in Fig. 12(a), (b), (c) and (d) for the angular velocities $\gamma=0$, 1, and 2. This is probably due to the fact that the optimized model in Fig. 12(e) gathers more materials in the left half part of the rotating thin plate so that when the angular 
velocity is small $(\gamma=0,1$, and 2$)$, the right half part of the rotating thin plate may control its first-order mode shape. Fig. 13 presents the first-order mode shapes of the optimized model in Fig. 12(e) at different angular velocities and vividly reveals that the above explanation is reasonable.
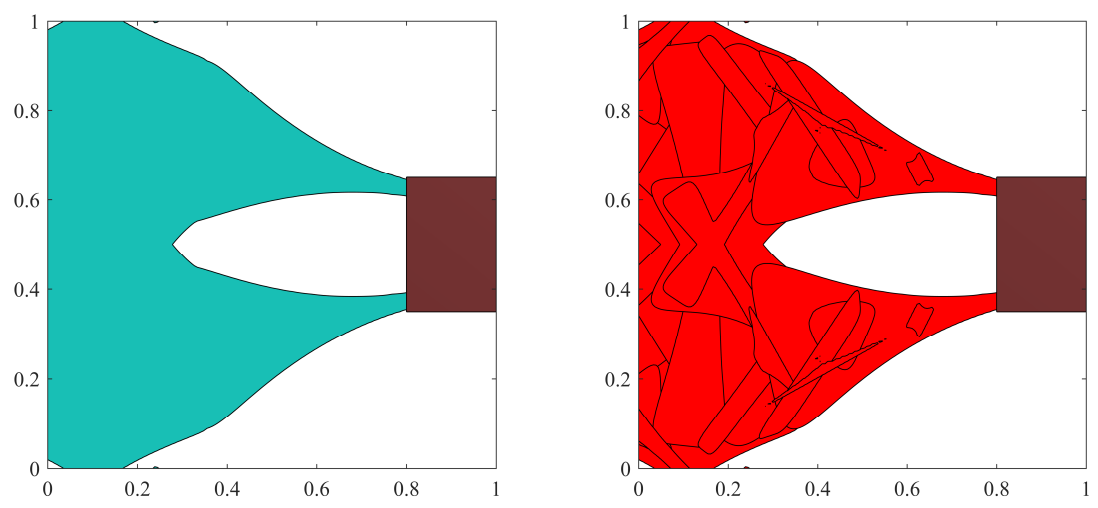

(a)
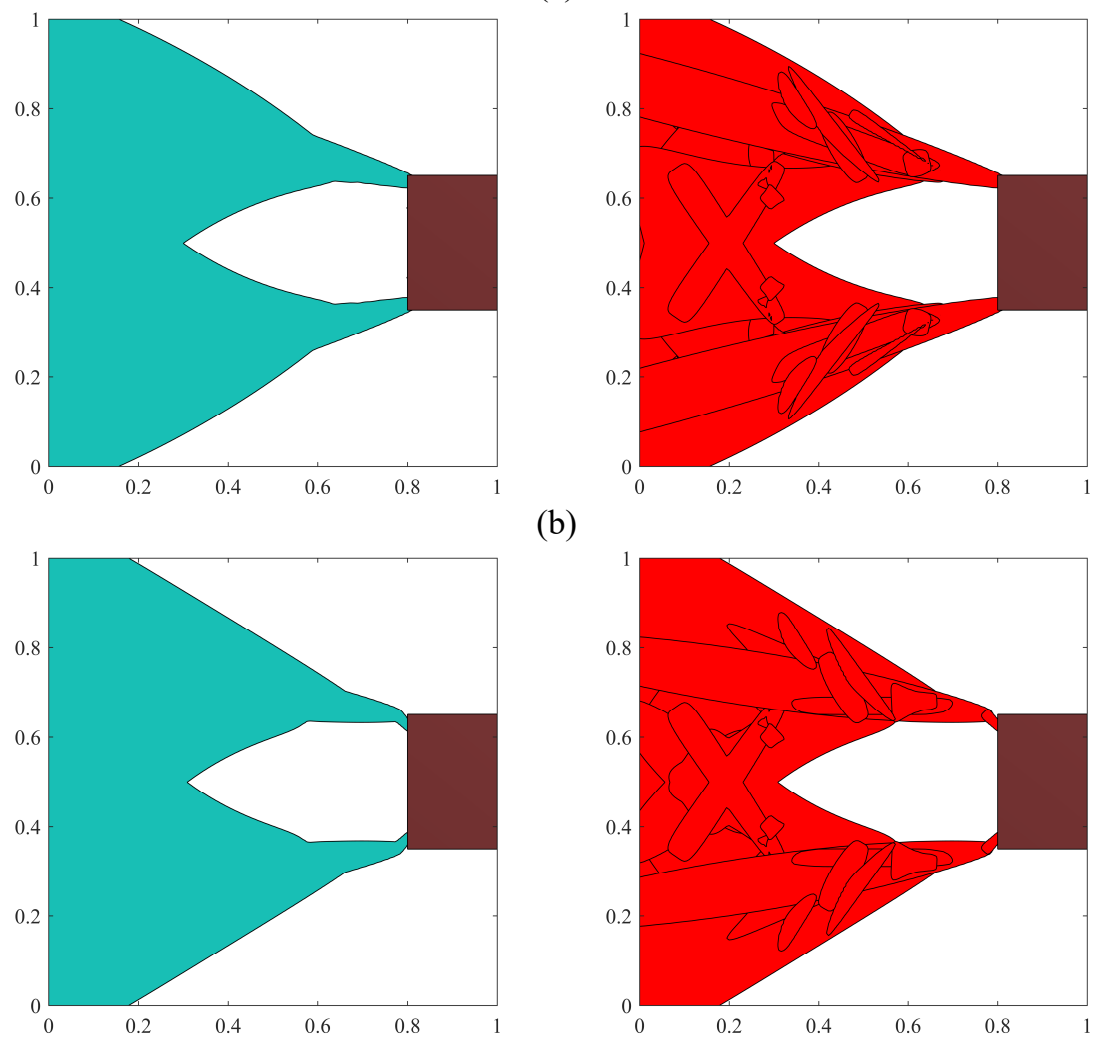

(b)
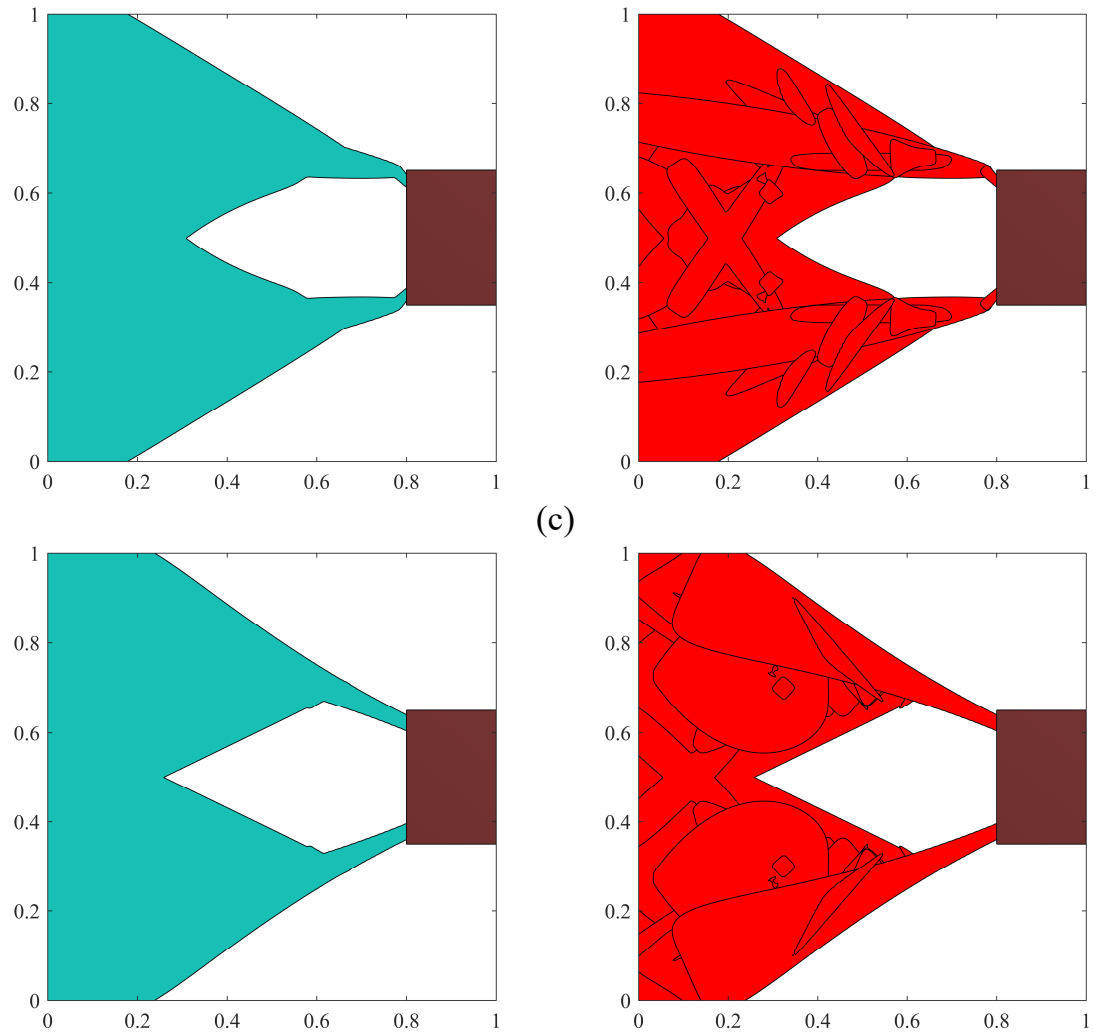

(c)

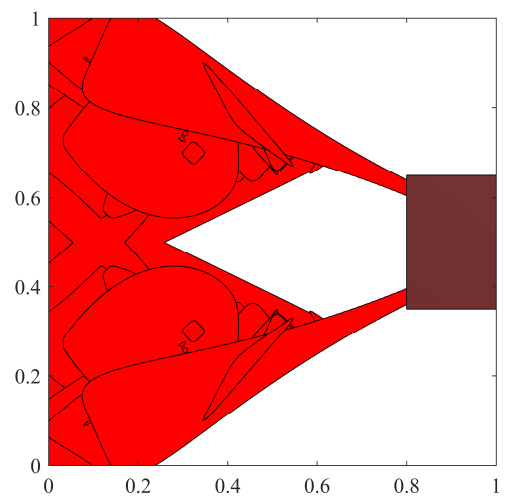

(d) 


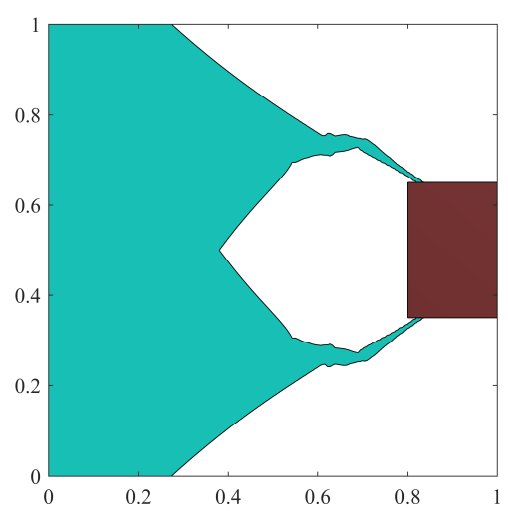

(e)

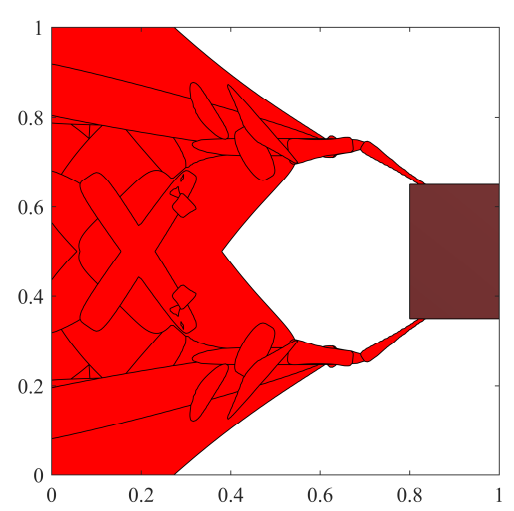

Fig. 14. Optimized results for maximizing the first eigenfrequency of the rotating thin plate with nonstructural mass: Case $2(\alpha=1, \beta=1, \mu=1000, \sigma=0)$. (a) $\gamma=0, \bar{\omega}_{1}=5.5880$; (b) $\gamma=1, \bar{\omega}_{1}=5.7111$; (c) $\gamma=2, \bar{\omega}_{1}=6.0632 ;$ (d) $\gamma=4, \bar{\omega}_{1}=7.2826 ;$ (e) $\gamma=8, \bar{\omega}_{1}=10.7958$.

Fig. 14 presents the optimized results for maximizing the first eigenfrequency of the rotating thin plate with the nonstructural mass in case 2 at five different angular velocities, i.e., $\gamma=0,1,2,4$, and 8. Different from case 1 in Fig. 12, the five optimized results in Fig. 14 all have the same topology, but different shapes. According to Table 2, the first eigenfrequencies of the optimized models in Fig. 14 increase from 2.3169, 2.5618, 3.1808, 4.8984 and 8.7772 of the unoptimized model to 5.5880, 5.7111, 6.0632, 7.2826 and 10.7958 , respectively for $\gamma=0,1,2,4$, and 8 . Table 2 also indicates that the five optimized models in Fig. 14 have all passed the cross-check. For the optimized model in Fig. 14(e), the same dynamic property as that in Fig. 12(e) is also demonstrated that the optimized model of a high angular velocity $(\gamma=8)$ behaves badly at low angular velocities $(\gamma=0,1$, and 2$)$. Here, the behavior means maximizing the first eigenfrequency. Hence, it is possible to reach the assertion that for maximizing the first eigenfrequency, one cannot obtain an exact optimized rotating thin plate for both low and high angular velocities.

\subsection{Maximizing the gap between two consecutive eigenfrequencies of the rotating thin plate with nonstructural mass}

The purpose of this subsection is to optimize a rotating thin plate for maximizing the gap between two consecutive eigenfrequencies. The rotating thin plate of concern has a length of $l_{1}=1 \mathrm{~m}$, a width of $l_{2}=0.2 \mathrm{~m}$, and a thickness of $l_{3}=0.001 \mathrm{~m}$. The material parameters of the rotating thin plate are also the same as those in Sections 6.2 and 6.3, i.e., Young's modulus $E=7.1 \times 10^{10} \mathrm{~Pa}$, Poison's ratio $v=0.3$, and mass density $\rho=2750$ $\mathrm{kg} / \mathrm{m}^{3}$. The subsection does not deal with the influence of the nonstructural mass on the optimized results, and, hence, focuses on one case of the nonstructural mass, i.e., $l_{1}^{*}=0.05 l_{1}, l_{2}^{*}=l_{2}$, and $\rho^{*}=\rho$. The radius of the rigid hub $R$ is also 0 . Likewise, the volume fraction $V_{\text {spec }}$ is set as 0.5 .

Before optimizing the rotating thin plate, the modal characteristic analysis of the unoptimized model is performed, as shown in Fig. 15. For better comparison, the unoptimized model has only a half part of materials evenly distributed in the design domain, i.e., $\rho_{e}=0.5$ for all the thin plate elements in the design domain. Fig. 15 shows 
the first four dimensionless eigenfrequencies and corresponding mode shapes of the rotating thin plate with an increase of the dimensionless angular velocity. It can be observed that the second and the third eigenfrequencies are cross with each other. When $\gamma \leq 7$, the second-order mode of the rotating thin plate is corresponding to the secondorder bending mode, while when $\gamma \geq 8$ and $\gamma \leq 10$, the second-order mode is corresponding to the first-order torsional mode. The first-order and fourth-order modes of the rotating thin plate are always corresponding to the first-order bending mode and third-order bending mode when $\gamma \leq 10$. From Fig. 15, it can also be seen that when $\gamma \leq 10$, the maximal gap between the second and third dimensionless eigenfrequencies is 8.9190, which is obtained from the case of $\gamma=0$. Therefore, the objective of this example is to maximize the gap between the second and the third eigenfrequencies of the rotating thin plate subject to a half part of materials in the design domain.

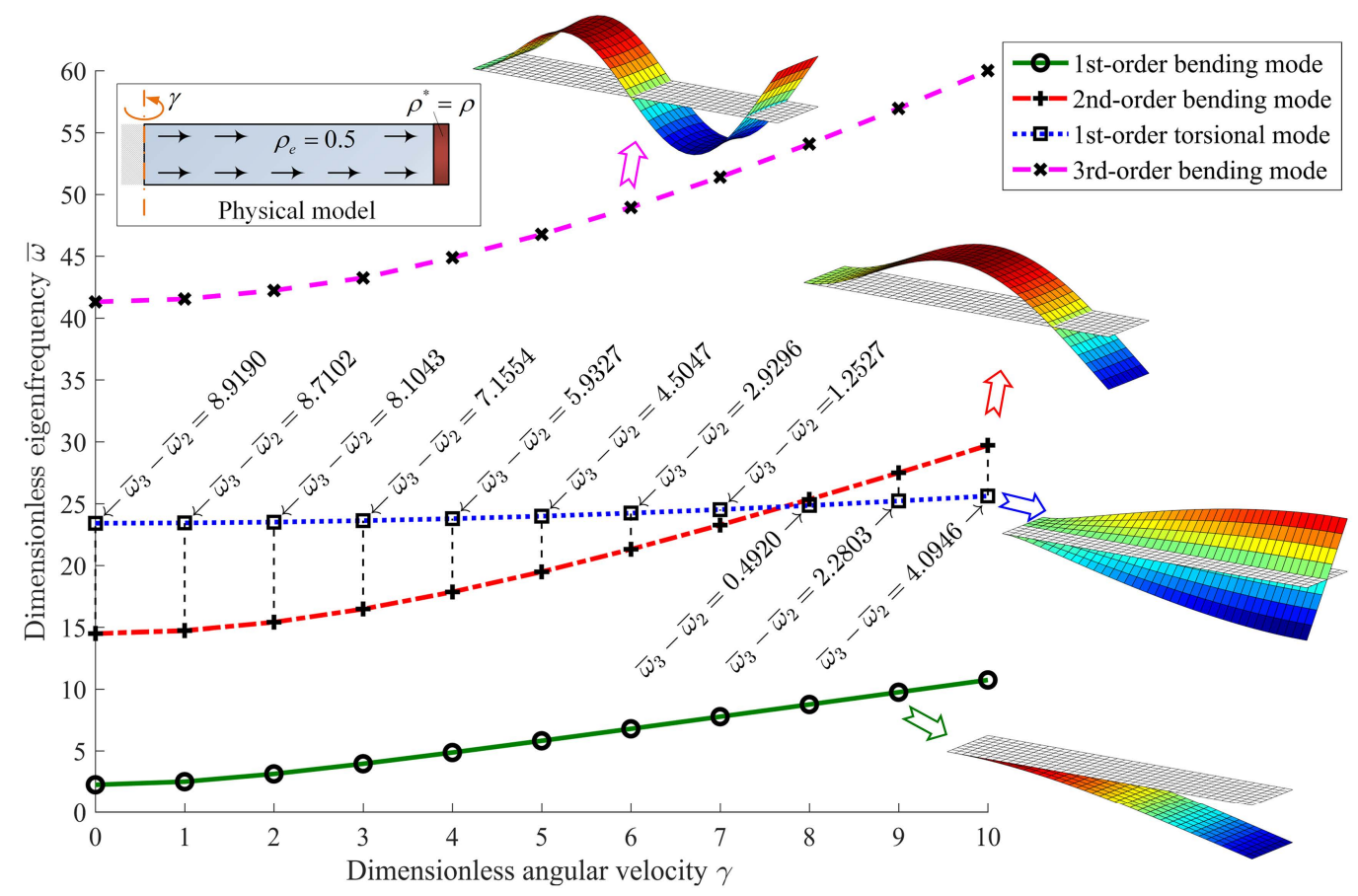

Fig. 15. The first four dimensionless eigenfrequencies versus dimensionless angular velocity for the unoptimized model $(\alpha=1, \beta=5, \mu=1000, \sigma=0)$.

Fig. 16 gives the initial configurations of the structural components. In this example, the symmetry property of the rotating thin plate is also taken into account so that 40 structural components are placed at the top half part of the thin plate. The number of design variables, hence, is 280 . To optimize the rotating thin plate in Fig. 16, six dimensionless angular velocities are considered, i.e., $\gamma=0,2,4,6,8$, and 10 . The optimized results are shown in Fig. 17, where the left column presents the contour plots and the right one gives the corresponding component plots of the optimized topologies. Fig. 17 also presents the gap between the second and the third dimensionless eigenfrequencies of the optimized rotating thin plate. For example, for the case of $\gamma=0$, the gap increases from 8.9190 of the unoptimized model in Fig. 15 to 39.0596 of the optimized model in Fig. 17(a). For the case of $\gamma=8$, the optimized model in Fig. 17(e) 
shows the largest increase in the gap, from 0.4920 of the unoptimized model in Fig. 15 to 39.6469 of the optimized one.

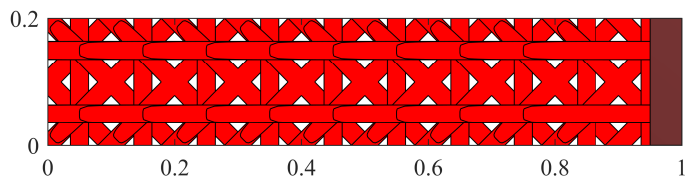

Fig. 16. Initial configurations of the components $(\alpha=1, \beta=5, \mu=1000, \sigma=0)$.
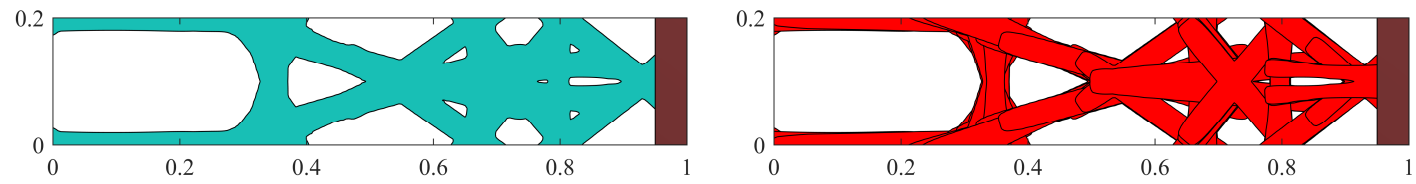

(a)
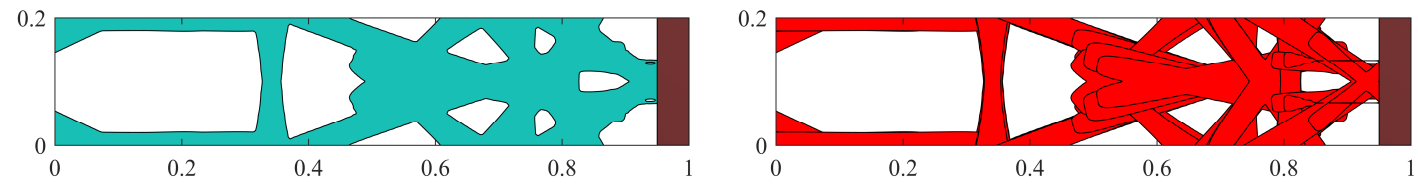

(b)
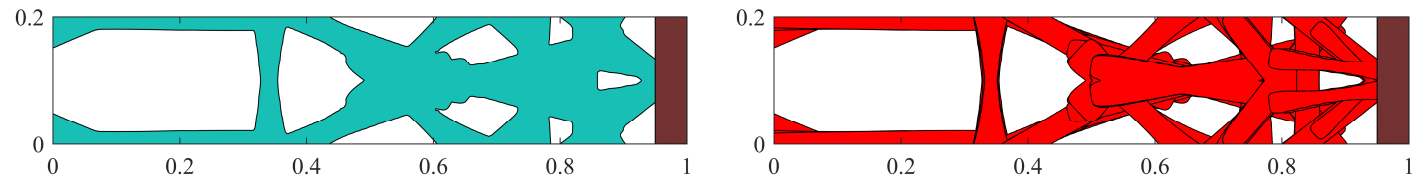

(c)
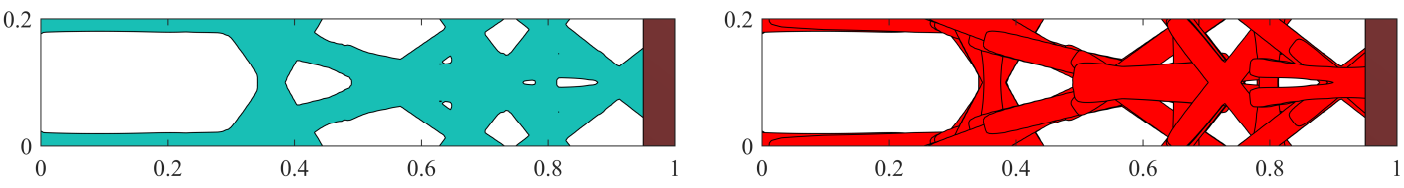

(d)
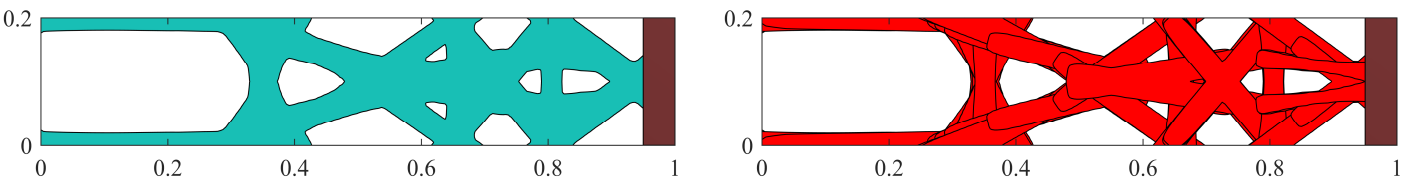

(e)
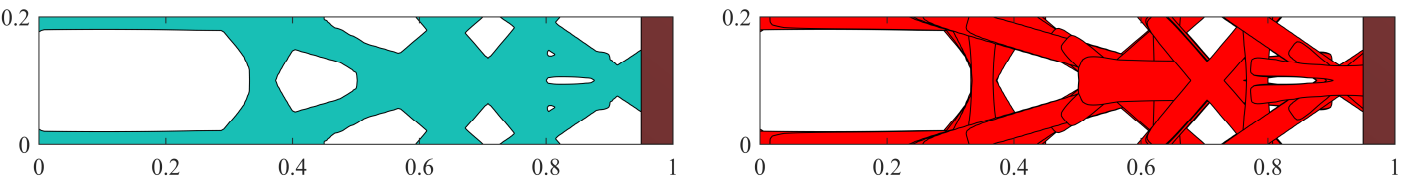

(f)

Fig. 17. Optimized results for maximizing the gap between the second and third eigenfrequencies of the rotating thin plate $(\alpha=1, \beta=5, \mu=1000, \sigma=0)$. (a) $\gamma=0, \bar{\omega}_{3}-\bar{\omega}_{2}=39.0596$; (b) $\gamma=2, \bar{\omega}_{3}-\bar{\omega}_{2}=40.9324$; (c) $\gamma=4, \quad \bar{\omega}_{3}-\bar{\omega}_{2}=40.8210$; (d) $\gamma=6, \bar{\omega}_{3}-\bar{\omega}_{2}=38.8361$; (e) $\gamma=8, \bar{\omega}_{3}-\bar{\omega}_{2}=39.6469$; (f) $\gamma=10$, $\bar{\omega}_{3}-\bar{\omega}_{2}=40.8569$.

From the optimized results in Fig. 17, it can be found that they have very similar topologies except for some details. Besides, the modal analysis results of the optimized models in Fig. 17 are only slightly different. Fig. 18 presents the first four dimensionless eigenfrequencies of the optimized models in Fig. 17 with an increase of angular velocity. Compared with the results in Fig. 15, Fig. 18 illustrates that the optimized models increase all the first four eigenfrequencies. For the unoptimized model, the second and 
the third eigenfrequency loci crossing phenomenon is observed, while for the optimized models in Fig. 18, the eigenfrequency loci crossing happens between the third and the fourth eigenfrequencies. The third-order and the fourth-order modes of the optimized models in Fig. 18 are corresponding to the third-order bending mode and the first-order torsional mode. Hence, it is easy to reach the assertion that maximizing the gap between the second and the third eigenfrequencies of the rotating thin plate is essentially equivalent to moving the eigenfrequency locus corresponding to the first-order torsional mode to a higher position. As a consequence, the phenomenon of eigenfrequency loci crossing happens between the third and the fourth eigenfrequencies. From Fig. 18(a) to Fig. 18(f), an interesting phenomenon can also be observed that with an increase of $\gamma$, the eigenfrequency loci crossing point of the optimized models also increases, but except for Fig. 18(b). For example, the eigenfrequency loci crossing points locate in the range of $\gamma \in[3,4]$ for Fig. 18(a), $\gamma \in[6,7]$ for Fig. 18(c), $\gamma \in[8,9]$ for Fig. 18(d), $\gamma \in[9,10]$ for Fig. 18(e), and $\gamma \in[10, \infty)$ for Fig. 18(f).

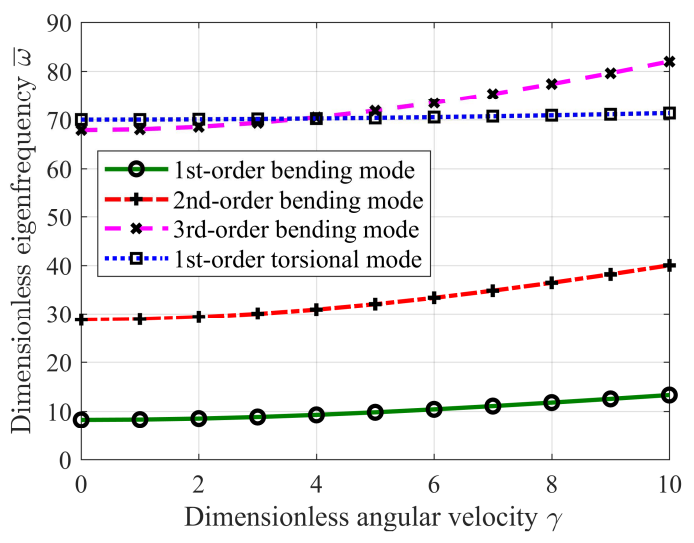

(a)

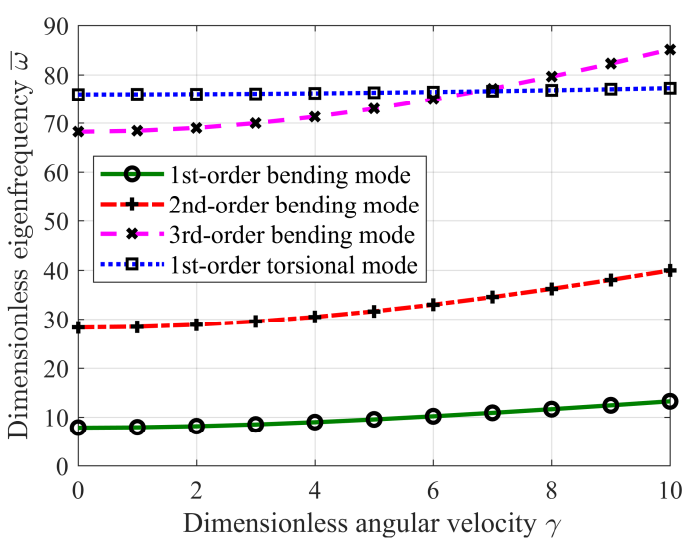

(c)

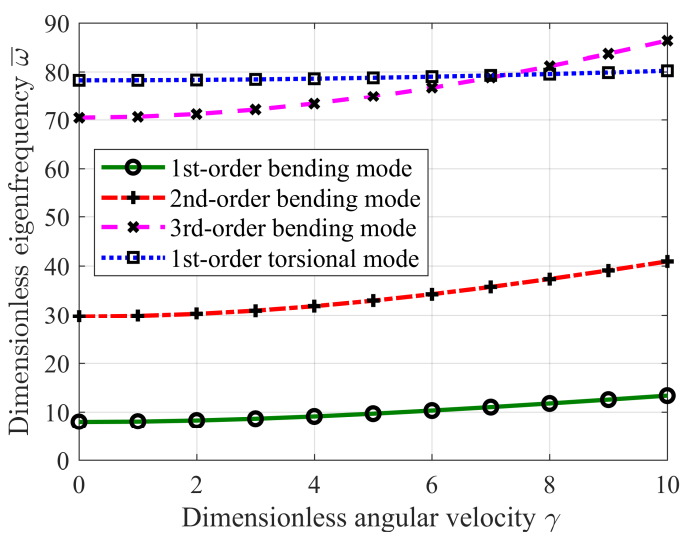

(b)

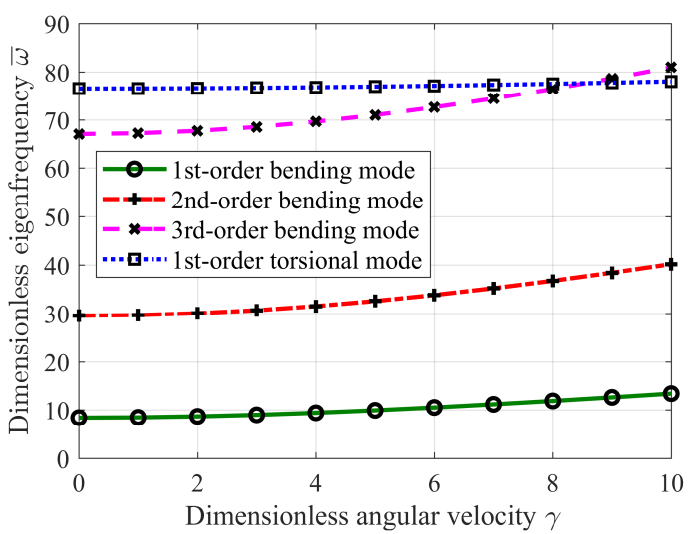

(d) 


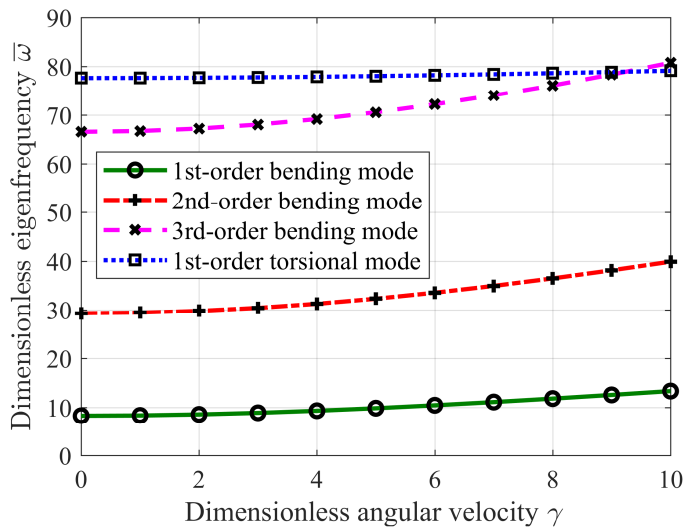

(e)

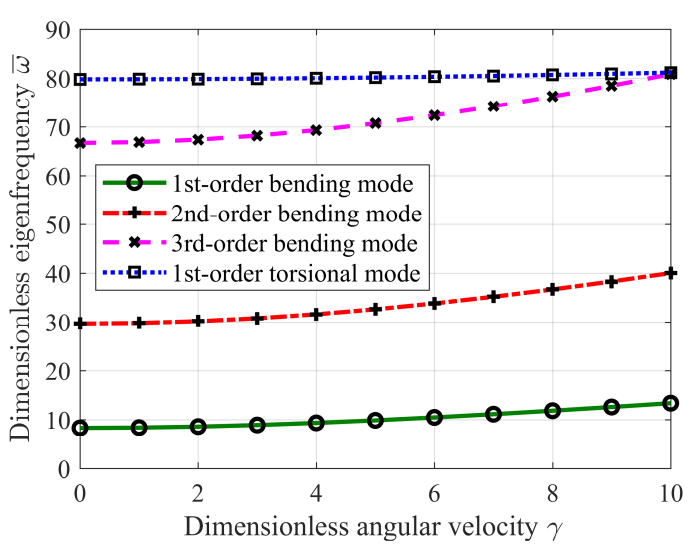

(f)

Fig. 18. The first four dimensionless eigenfrequencies versus dimensionless angular velocity for the optimized rotating thin plates in Fig. $17(\alpha=1, \beta=5, \mu=1000, \sigma=0)$. (a) Optimized model with $\gamma=0$ in Fig. 17(a); (b) optimized model with $\gamma=2$ in Fig. 17(b); (c) optimized model with $\gamma=4$ in Fig. 17(c); (d) optimized model with $\gamma=6$ in Fig. 17(d); (e) optimized model with $\gamma=8$ in Fig. 17(e); (f) optimized model with $\gamma=10$ in Fig. 17(f).

\section{Conclusions}

The paper presents a new efficient and explicit topology optimization approach for the eigenfrequencies of a rotating thin plate. In order to accurately describe the dynamics of the rotating thin plate and to take the membrane deformation of the thin plate into consideration, the approach uses the thin plate elements of the absolute nodal coordinate formulation (ANCF) to model the rotating thin plate. When performing the modal characteristic analysis of the rotating thin plate at a particular static equilibrium configuration and a prescribed angular velocity, the approach employs linearization techniques. To reduce the computation cost, the approach embeds the moving morphable components (MMC) method, which uses the geometrical parameters, positions and the orientation of a number of thickness-varying structural components as the design variables to explicitly describe the topology of the rotating thin plate and to reduce the number of the design variables. Furthermore, the approach adopts the penalization for the mass and stiffness matrices of the thin plate elements of ANCF carefully so as to remove the localized modes of the rotating thin plate in the lowdensity areas. To complete the optimization for eigenfrequencies, the paper also gives the analytical formulae of the sensitivities of a simple eigenfrequency and multiply repeated eigenfrequencies with respect to a design variable.

The paper validates the proposed topology optimization approach via two benchmark examples and two optimization examples. The two benchmark examples show that the optimization model of the rotating thin plate and the new topology optimization approach are both correct and effective. The two optimization examples take two different objectives and present the influence of the nonstructural mass and angular velocity on the optimized results, including some interesting phenomena observed in the topology optimization. The four examples reveal that the linearization techniques can effectively and efficiently solve the topology optimization problem for eigenfrequencies so as to facilitate the design of the rotating thin plate with linear 
vibration.

\section{Acknowledgments}

This work was supported in part by the National Natural Science Foundation of China under Grants 11722216 and 11832005.

\section{Appendix}

The terms in the dimensionless form of the dynamic equation (24) and the eigenvalue problem (25) can be expressed as follows

$$
\begin{aligned}
& \overline{\mathbf{M}}=\sum_{e=1}^{N E 1} \mathbf{B}_{e}^{\mathrm{T}} \overline{\mathbf{M}}_{e} \mathbf{B}_{e}+\sum_{e=1}^{N E 2} \mathbf{B}_{e}^{\mathrm{T}} \overline{\mathbf{M}}_{e}^{*} \mathbf{B}_{e}, \\
& \overline{\mathbf{F}}(\overline{\mathbf{q}})=\sum_{e=1}^{N E} \mathbf{B}_{e}^{\mathrm{T}} \overline{\mathbf{F}}_{e}, \\
& \overline{\mathbf{Q}}(\overline{\mathbf{q}})=\sum_{e=1}^{N E 1} \mathbf{B}_{e}^{\mathrm{T}} \overline{\mathbf{Q}}_{e}+\sum_{e=1}^{N E 2} \mathbf{B}_{e}^{\mathrm{T}} \overline{\mathbf{Q}}_{e}^{*}, \\
& \overline{\mathbf{K}}(\overline{\mathbf{q}})=\sum_{e=1}^{N E} \mathbf{B}_{e}^{\mathrm{T}} \frac{\partial \overline{\mathbf{F}}_{e}}{\partial \overline{\mathbf{Q}}} \mathbf{B}_{e}-\sum_{e=1}^{N E 1} \mathbf{B}_{e}^{\mathrm{T}} \frac{\partial \overline{\mathbf{Q}}_{e}}{\partial \overline{\mathbf{e}}} \mathbf{B}_{e}-\sum_{e=1}^{N E 2} \mathbf{B}_{e}^{\mathrm{T}} \frac{\partial \overline{\mathbf{Q}}_{e}^{*}}{\partial \overline{\mathbf{e}}} \mathbf{B}_{e},
\end{aligned}
$$

where

$$
\begin{aligned}
& \overline{\mathbf{M}}_{e}=\int_{0}^{1} \int_{0}^{1} \overline{\mathbf{S}}(\xi, \eta)^{\mathrm{T}} \overline{\mathbf{S}}(\xi, \eta) \mathrm{d} \xi \mathrm{d} \eta, \\
& \overline{\mathbf{M}}_{e}^{*}=\alpha \int_{0}^{1} \overline{\mathbf{S}}(1, \eta)^{\mathrm{T}} \overline{\mathbf{S}}(1, \eta) \mathrm{d} \eta, \\
& \overline{\mathbf{F}}_{e}=\overline{\mathbf{F}}_{e}^{\varepsilon}+\overline{\mathbf{F}}_{e}^{\kappa}=12 \mu^{2} \int_{0}^{1} \int_{0}^{1}\left(\frac{\partial \overline{\boldsymbol{\varepsilon}}}{\partial \overline{\mathbf{e}}}\right)^{\mathrm{T}} \mathbf{E} \overline{\boldsymbol{\varepsilon}} \mathrm{d} \xi \mathrm{d} \eta+\int_{0}^{1} \int_{0}^{1}\left(\frac{\partial \overline{\mathbf{K}}}{\partial \overline{\mathbf{e}}}\right)^{\mathrm{T}} \mathbf{E} \overline{\mathbf{K}} \mathrm{d} \xi \mathrm{d} \eta, \\
& \overline{\mathbf{Q}}_{e}=\gamma^{2} \int_{0}^{1} \int_{0}^{1} \overline{\mathbf{S}}(\xi, \eta)^{\mathrm{T}} \overline{\tilde{\mathbf{S}}}(\xi, \eta) \mathrm{d} \xi \mathrm{d} \eta \overline{\mathbf{e}}+\sigma \gamma^{2} \int_{0}^{1} \int_{0}^{1} \overline{\mathbf{S}}(\xi, \eta)^{\mathrm{T}} \mathrm{d} \xi \mathrm{d} \eta, \\
& \overline{\mathbf{Q}}_{e}^{*}=\alpha \gamma^{2} \int_{0}^{1} \int_{0}^{1} \overline{\mathbf{S}}(\xi, \eta)^{\mathrm{T}} \overline{\tilde{\mathbf{S}}}(\xi, \eta) \mathrm{d} \xi \mathrm{d} \eta \overline{\mathbf{e}}+\alpha \sigma \gamma^{2} \int_{0}^{1} \int_{0}^{1} \overline{\mathbf{S}}_{1}(\xi, \eta)^{\mathrm{T}} \mathrm{d} \xi \mathrm{d} \eta,
\end{aligned}
$$

with

$$
\begin{aligned}
\overline{\mathbf{q}} & =\sum_{e=1}^{N E} \mathbf{B}_{e}^{\mathrm{T}} \mathbf{\mathbf { e }} \\
\overline{\mathbf{e}} & =\frac{1}{l_{1}}\left[\begin{array}{lllllll}
\mathbf{r}_{1}^{\mathrm{T}} & l_{1} \mathbf{r}_{1, x}^{\mathrm{T}} & l_{1} \mathbf{r}_{1, y}^{\mathrm{T}} & \cdots & \mathbf{r}_{4}^{\mathrm{T}} & l_{1} \mathbf{r}_{4, x}^{\mathrm{T}} & l_{1} \mathbf{r}_{4, y}^{\mathrm{T}}
\end{array}\right]^{\mathrm{T}}, \\
\overline{\mathbf{S}} & =\left[\begin{array}{lllllll}
S_{1} \mathbf{I} & \frac{S_{2}}{l_{1}} \mathbf{I} & \frac{S_{3}}{l_{1}} \mathbf{I} & \cdots & S_{10} \mathbf{I} & \frac{S_{11}}{l_{1}} \mathbf{I} & \frac{S_{12}}{l_{1}} \mathbf{I}
\end{array}\right], \\
\overline{\tilde{\mathbf{S}}} & =\left[\begin{array}{lllllll}
S_{1} \tilde{\mathbf{I}} & \frac{S_{2}}{l_{1}} \tilde{\mathbf{I}} & \frac{S_{3}}{l_{1}} \tilde{\mathbf{I}} & \cdots & S_{10} \tilde{\mathbf{I}} & \frac{S_{11}}{l_{1}} \tilde{\mathbf{I}} & \frac{S_{12}}{l_{1}} \tilde{\mathbf{I}}
\end{array}\right], \\
\overline{\mathbf{S}}_{1} & =\left[\begin{array}{lllllll}
S_{1} \mathbf{I}_{1} & \frac{S_{2}}{l_{1}} \mathbf{I}_{1} & \frac{S_{3}}{l_{1}} \mathbf{I}_{1} & \cdots & S_{10} \mathbf{I}_{1} & \frac{S_{11}}{l_{1}} \mathbf{I}_{1} & \frac{S_{12}}{l_{1}} \mathbf{I}_{1}
\end{array}\right],
\end{aligned}
$$




$$
\begin{aligned}
& \overline{\mathbf{r}}=\overline{\mathbf{S}}(\xi, \eta) \overline{\mathbf{e}}=\frac{1}{l_{1}} \mathbf{S}(\xi, \eta) \mathbf{e}=\frac{\mathbf{r}}{l_{1}}, \\
& \overline{\boldsymbol{\varepsilon}}=\left[\begin{array}{lll}
\frac{1}{2}\left(N X^{2} \cdot \overline{\mathbf{r}}_{, \xi}^{\mathrm{T}} \overline{\mathbf{r}}_{, \xi}-1\right) & \frac{1}{2}\left(N Y^{2} \cdot \beta^{2} \cdot \overline{\mathbf{r}}_{, \eta}^{\mathrm{T}} \overline{\mathbf{r}}_{, \eta}-1\right) & N X \cdot N Y \cdot \beta \cdot \overline{\mathbf{r}}_{, \xi}^{\mathrm{T}} \overline{\mathbf{r}}_{, \eta}
\end{array}\right]^{\mathrm{T}}, \\
& \overline{\mathbf{\kappa}}=\left[\begin{array}{lll}
\frac{N X^{2} \cdot \overline{\mathbf{r}}_{, \xi \xi}^{\mathrm{T}} \overline{\mathbf{n}}}{\|\overline{\mathbf{n}}\|^{3}} & \frac{N Y^{2} \cdot \beta^{2} \cdot \overline{\mathbf{r}}_{, \eta \eta}^{\mathrm{T}} \overline{\mathbf{n}}}{\|\overline{\mathbf{n}}\|^{3}} & \frac{N X \cdot N Y \cdot \beta \cdot \overline{\mathbf{r}}_{, \xi \eta}^{\mathrm{T}} \overline{\mathbf{n}}}{\|\overline{\mathbf{n}}\|^{3}}
\end{array}\right]^{\mathrm{T}}, \\
& \overline{\mathbf{n}}=N X \cdot N Y \cdot \beta \cdot\left(\overline{\mathbf{r}}_{, \xi} \times \overline{\mathbf{r}}_{, \eta}\right) .
\end{aligned}
$$

\section{References}

[1] X.S. Zhang, D.G. Zhang, S.J. Chen, J.Z. Hong, Modal characteristics of a rotating flexible beam with a concentrated mass based on the absolute nodal coordinate formulation, Nonlinear Dynamics 88(1) (2017) 61-77.

[2] M. Rafiee, F. Nitzsche, M. Labrosse, Dynamics, vibration and control of rotating composite beams and blades: a critical review, Thin-Walled Structures 119 (2017) 795-819.

[3] J. Zhao, Q. Tian, H.Y. Hu, Modal analysis of a rotating thin plate via absolute nodal coordinate formulation, Journal of Computational and Nonlinear Dynamics 6(4) (2011) 041013.

[4] J. Liu, Q. Li, S. Liu, L. Tong, Dynamic topology optimization design of rotating beam cross-section with gyroscopic effects, Structural and Multidisciplinary Optimization 58(4) (2018) 1467-1487.

[5] M. Berzeri, A.A. Shabana, Study of the centrifugal stiffening effect using the finite element absolute nodal coordinate formulation, Multibody System Dynamics 7 (2002) 367-387.

[6] D. García-Vallejo, H. Sugiyama, A.A. Shabana, Finite element analysis of the geometric stiffening effect. Part 2: non-linear elasticity, Proceedings of the Institution of Mechanical Engineers, Part K: Journal of Multi-Body Dynamics 219(2) (2005) 203-211.

[7] L.G. Maqueda, O.A. Bauchau, A.A. Shabana, Effect of the centrifugal forces on the finite element eigenvalue solution of a rotating blade: a comparative study, Multibody System Dynamics 19(3) (2008) 281-302.

[8] H.H. Yoo, C. Pierre, Modal characteristic of a rotating rectangular cantilever plate, Journal of Sound and Vibration 259(1) (2003) 81-96.

[9] H.H. Yoo, S.K. Kim, D.J. Inman, Modal analysis of rotating composite cantilever plates, Journal of Sound and Vibration 258(2) (2002) 233-246.

[10] A. Karmakar, P.K. Sinha, Finite element free vibration analysis of rotating laminated composite pretwisted cantilever plates, Journal of Reinforced Plastics and Composites 16(16) (1997) 14611491.

[11] V. Ramamurti, R. Kielb, Natural frequencies of twisted rotating plates, Journal of Sound and Vibration 97(3) (1984) 429-449.

[12] M.A. Dokainish, S. Rawtani, Vibration analysis of rotating cantilever plates, International Journal for Numerical Methods in Engineering 3(2) (1971) 233-248.

[13] A.A. Shabana, An absolute nodal coordinates formulation for the large rotation and deformation analysis of flexible bodies, Report. Report No. MBS96-1-UIC, University of Illinois at Chicago, 1996.

[14] A.A. Shabana, On the definition of the natural frequency of oscillations in nonlinear large rotation problems, Journal of Sound and Vibration 329(15) (2010) 3171-3181. 
[15] A.A. Shabana, M.H. Zaher, A.M. Recuero, C. Rathod, Study of nonlinear system stability using eigenvalue analysis: gyroscopic motion, Journal of Sound and Vibration 330(24) (2011) 60066022.

[16] C. Yang, D. Cao, Z. Zhao, Z. Zhang, G. Ren, A direct eigenanalysis of multibody system in equilibrium, Journal of Applied Mathematics 2012 (2012) 1-12.

[17] I. Kosaka, C.C. Swan, A symmetry reduction method for continuum structural topology optimization, Computers and Structures 70(1) (1999) 47-61.

[18] Z. Ma, N. Kikuchi, H. Cheng, Topological design for vibrating structures, Computer Methods in Applied Mechanics and Engineering 121(1) (1995) 259-280.

[19] J.S. Jensen, N.L. Pedersen, On maximal eigenfrequency separation in two-material structures: the 1D and 2D scalar cases, Journal of Sound and Vibration 289(4-5) (2006) 967-986.

[20] N.L. Pedersen, Maximization of eigenvalues using topology optimization, Structural and Multidisciplinary Optimization 20(1) (2000) 2-11.

[21] F. Ferrari, B.S. Lazarov, O. Sigmund, Eigenvalue topology optimization via efficient multilevel solution of the frequency response, International Journal for Numerical Methods in Engineering 115(7) (2018) 872-892.

[22] O. Sigmund, K. Maute, Topology optimization approaches: a comparative review, Structural and Multidisciplinary Optimization 48(6) (2013) 1031-1055.

[23] M.P. Bendsøe, N. Kikuchi, Generating optimal topologies in structural design using a homogenization method, Computer Methods in Applied Mechanics and Engineering 71(2) (1988) 197-224.

[24] Q. Xia, T. Shi, M.Y. Wang, A level set based shape and topology optimization method for maximizing the simple or repeated first eigenvalue of structure vibration, Structural and Multidisciplinary Optimization 43(4) (2011) 473-485.

[25] X. Guo, W. Zhang, W. Zhong, Doing topology optimization explicitly and geometrically-a new moving morphable components based framework, Journal of Applied Mechanics 81(8) (2014) 081009 .

[26] W. Zhang, D. Li, J. Yuan, J. Song, X. Guo, A new three-dimensional topology optimization method based on moving morphable components (MMCs), Computational Mechanics 69(4) (2017) 647665 .

[27] W. Zhang, J. Yuan, J. Zhang, X. Guo, A new topology optimization approach based on moving morphable components (MMC) and the ersatz material model, Structural and Multidisciplinary Optimization 53(6) (2016) 1243-1260.

[28] J.L. Sun, Q. Tian, H.Y. Hu, Topology optimization of a three-dimensional flexible multibody system via moving morphable components, Journal of Computational and Nonlinear Dynamics 13(2) (2018) 021010.

[29] C.R. Thomsen, F. Wang, O. Sigmund, Buckling strength topology optimization of 2D periodic materials based on linearized bifurcation analysis, Computer Methods in Applied Mechanics and Engineering 339 (2018) 115-136.

[30] D. Tcherniak, Topology optimization of resonating structures using SIMP method, International Journal for Numerical Methods in Engineering 54(11) (2002) 1605-1622.

[31] A.P. Seyranian, E. Lund, N. Olhoff, Multiple eigenvalues in structural optimization problems, Structural Optimization 8(4) (1994) 207-227.

[32] X. Chen, H. Qi, L. Qi, K. Teo, Smooth convex approximation to the maximum eigenvalue function, 
$30(2)(2004) 253-270$.

[33] J. Gravesen, A. Evgrafov, D.M. Nguyen, On the sensitivities of multiple eigenvalues, Structural and Multidisciplinary Optimization 44(4) (2011) 583-587.

[34] A.J. Torii, J.R.D. Faria, Structural optimization considering smallest magnitude eigenvalues: a smooth approximation, Journal of the Brazilian Society of Mechanical Sciences and Engineering 39(5) (2017) 1745-1754.

[35] K. Dufva, A.A. Shabana, Analysis of thin plate structures using the absolute nodal coordinate formulation, Proceedings of the Institution of Mechanical Engineers, Part K: Journal of MultiBody Dynamics 219(4) (2005) 345-355.

[36] K.J. Bathe, The subspace iteration method - revisited, Computers \& Structures 126 (2013) 177183.

[37] K. Bathe, S. Ramaswamy, An accelerated subspace iteration method, Computer Methods in Applied Mechanics and Engineering 23(3) (1980) 313-331.

[38] W. Zhang, J.H. Zhou, Y. Zhu, X. Guo, Structural complexity control in topology optimization via moving morphable component (MMC) approach, Structural and Multidisciplinary Optimization 56(3) (2017) 535-552.

[39] J.L. Sun, Q. Tian, H.Y. Hu, N.L. Pedersen, Topology optimization of a flexible multibody system with variable-length bodies described by ALE-ANCF, Nonlinear Dynamics 93(2) (2018) 413-441.

[40] X. Guo, K. Zhao, M.Y. Wang, A new approach for simultaneous shape and topology optimization based on dynamic implicit surface function, Control and Cybernetics 34(1) (2005) 255-282.

[41] M.Y. Wang, X.M. Wang, D.M. Guo, A level set method for structural topology optimization, Computer Methods in Applied Mechanics and Engineering 192 (2003) 227-246.

[42] Y. Zhou, W. Zhang, J. Zhu, Z. Xu, Feature-driven topology optimization method with signed distance function, Computer Methods in Applied Mechanics and Engineering 310 (2016) 1-32.

[43] W. Zhang, J. Chen, X. Zhu, J. Zhou, D. Xue, X. Lei, X. Guo, Explicit three dimensional topology optimization via moving morphable void (MMV) approach, Computer Methods in Applied Mechanics and Engineering 322 (2017) 590-614.

[44] P. Pedersen, N.L. Pedersen, Interpolation/penalization applied for strength design of 3D thermoelastic structures, Structural and Multidisciplinary Optimization 45(6) (2012) 773-786.

[45] K. Svanberg, The method of moving asymptotes - a new method for structural optimization, International Journal for Numerical Methods in Engineering 24(2) (1987) 359-373.

[46] D.A. Tortorelli, P. Michaleris, Design sensitivity analysis: overview and review, Inverse Problems in Engineering 1(1) (1994) 71-105.

[47] R. Xue, C. Liu, W. Zhang, Y. Zhu, S. Tang, Z. Du, X. Guo, Explicit structural topology optimization under finite deformation via moving morphable void (MMV) approach, Computer Methods in Applied Mechanics and Engineering 344 (2019) 798-818. 